\title{
Merced Peak Quadrangle, Central Sierra Nevada, California - Analytic Data
}

GEOLOGICALSURVEY PROFESSIONAL PAPER 1170-D

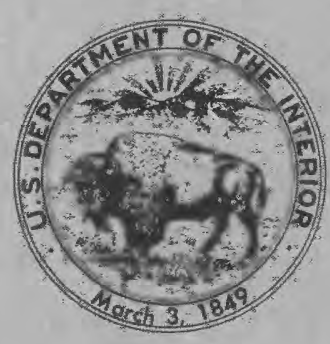




\section{Merced Peak Quadrangle, Central Sierra Nevada, California-Analytic Data}

By DALLAS L. PECK and GERALD K. VAN KOOTEN

SHORTER GONTRIBUTIONS TO GENERAL GEOLOGY

GEOLOGICALSURVEY PROFESSIONALA PER 1170-D

Chemical, semiquantitative spectrographic, and modal analyses and potassium-argon, uranium-lead, and rubidium-strontium age determinations on plutonic, metavolcanic, and volcanic rocks supplement U.S. Geological Survey Geologic Quadrangle Map GQ-1531

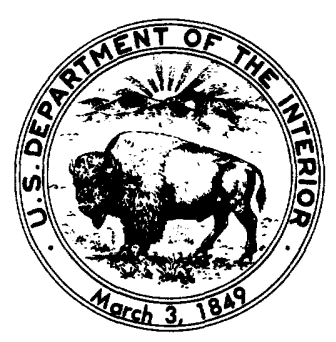

UNITED STATES GOVERNMENT PRINTING OFFICE, WASHINGTON:1983 
UNITED STATES DEPARTMENT OF THE INTERIOR

JAMES G. WATT, Secretary

GEOLOGICAL SURVEY

Dallas L. Peck, Director

For sale by the Distribution Branch, U.S. Geological Survey, 604 South Pickett Street, Alexandria, VA 22304 


\section{CONTENTS}

$\begin{array}{lr}\text { Abstract } & \text { Page } \\ \text { Introduction } & 1 \\ \text { General geology } & 1 \\ \text { Analytic data } & \\ \text { References cited } & \end{array}$

\section{ILLUSTRATIONS}

Figures 1-8. Simplified bedrock geologic maps of the Merced Peak quadrangle, showing:

1. Locations of chemically analyzed and isotopically dated rock samples

2. Steeply inclined regional joints

3. Locations of modally analyzed plutonic rock samples _

4. Quartz in volume percent ___- 20

5. Potassium feldspar in volume percent

6. Plagioclase in volume percent

7. Mafic minerals in volume percent

8. Specific gravity

9. Plots of modes of granitic rocks

10. Plots of norms of plutonic and metavolcanic rocks

\section{TABLES}

TABLE 1. Rubidium and strontium determinations on metavolcanic rocks

Page

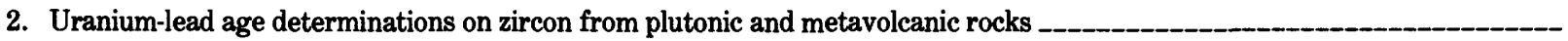

3. Potassium-argon age determinations on minerals from granitic and volcanic rocks _-__-__-__-_-

4. Chemical and spectrographic analyses, norms, and modes of representative igneous rocks

5. Modes, specific gravity, and location of plutonic rocks 

SHORTER CONTRIBUTIONS TO GENERAL GEOLOGY

\title{
MERCED PEAK QUADRANGLE, GENTRAL SIERRA NEVADA, CALIFORNIA - ANAL YTIC DATA
}

\author{
By Dallas L. Peck and Gerald K. Van KoOten ${ }^{1}$
}

\begin{abstract}
A variety of plutonic, metamorphic, and volcanic rocks and glacial deposits underlie the Merced Peak quadrangle, which spans the southeastern margin of Yosemite National Park. More than 800 rock samples were collected during geologic mapping of the area, of which 258 samples of plutonic rocks were analyzed modally and their specific gravities determined. The volume percentages of quartz, potassium feldspar, plagioclase, and mafic minerals and the bulk specific gravities are plotted on a simplified geologic base map and contoured where the data permit. Quartz, potassium feldspar, and plagioclase, calculated to 100 percent, are plotted on triangular diagrams. The modal analyses show that the compositions of individual plutons are predominantly granite and granodiorite but range from leucogranite to tonalite and quartz diorite. Chemical and spectrographic analyses of 41 plutonic rocks, 15 metavolcanic rocks, and 11 Pliocene volcanic rocks are tabulated, and normative quartz, orthoclase, and plagioclase of the plutonic and metavolcanic rocks are plotted on a triangular diagram. The metavolcanic rocks are primarily rhyodacite but range from rhyolite to andesite. The Pliocene volcanic rocks are predominantly trachybasalt but include several flows and volcanic necks of ultrapotassic mafic phonolite. Data on ages determined by the potassiumargon, uranium-lead, and rubidium-strontium methods on some plutonic, metavolcanic, and volcanic rocks are tabulated. A map of conspicuous, steeply dipping regional joints is included.
\end{abstract}

\section{INTRODUCTION}

The Merced Peak quadrangle includes an area of about $620 \mathrm{~km}^{2}$ of the Sierra Nevada batholith $10-40 \mathrm{~km}$ southeast and east of Yosemite Valley. The quadrangle is nearly bisected by the southeastern boundary of Yosemite National Park, which for the most part coincides in the area with the divide between the drainage basins of the Merced and San Joaquin Rivers. Altitudes range from 6,100 to 13,100 feet (about 2,000 to 4,000 m), and much of the area is at or near local timberline. Roads are limited to the southern margin, but a network of trails provides access to all parts of the area.

This paper supplements the "Geologic Map of the Merced Peak Quadrangle, Central Sierra Nevada, California" (Peck, 1980), by providing analytic data on the plutonic, metamorphic, and volcanic rocks of the

${ }^{1}$ Arco Oil and Gas, Geothermal Projects, P.O. Box 2819, Dallas, TX 75221. quadrangle. It is part of a continuing study of the bedrock geology of the central Sierra Nevada batholith reported earlier by Bateman and others (1963), Bateman and Wahrhaftig (1966), and Bateman and Eaton (1967). A preliminary version of the geologic map of the Merced Peak quadrangle was published in uncolored form in 1964 (Peck, D. L., 1964). The bedrock geology of the northwestern part of the quadrangle was mapped and described by Calkins (1930), and the glacial deposits have been studied by Matthes $(1930 ; 1960)$ and Birman (1964). Nokleberg $(1970 ; 1981)$ mapped the Strawberry Mine area in detail as part of this project, and his mapping is incorporated in figure 1. Geologic maps of adjacent quadrangles have been published by Huber and Rinehart (1965), Kistler (1966a), Huber (1968), Bateman and others (1971), and Kistler (1973). Supplementary geologic and analytic reports include Kistler (1966b), Huber and Rinehart (1967), Bateman and Lockwood (1976), and Kistler (1974). Isotopic U-Pb ages of zircons from several of the plutonic units of the Merced Peak quadrangle, as well as from many other plutons of the central Sierra Nevada, have been reported recently by Stern and others (1981). Plutonic rocks in this report are classified according to the recommendations of the International Union of Geological Sciences (Streckeisen and others, 1973) as shown in figure $9 A$.

\section{GENERAL GEOLOGY}

The quadrangle is underlain chiefly by plutonic and metavolcanic rocks, as shown in the simplified bedrock geologic map (fig. 1) and described in the text of the geologic map of the Merced Peak quadrangle (Peck, 1980). Metasedimentary rocks are exposed only in a few localities. The rocks are predominantly metaquartzite but include less abundant quartz-biotite hornfels, quartzplagioclase hornfels, tactite, and marble. A fossil from the Strawberry Mine area, identified by R.W. Imlay (oral commun., 1977) as Inoceramus pseudomytiloides(?) of Early Jurassic age, indicates that the beds are cor- 


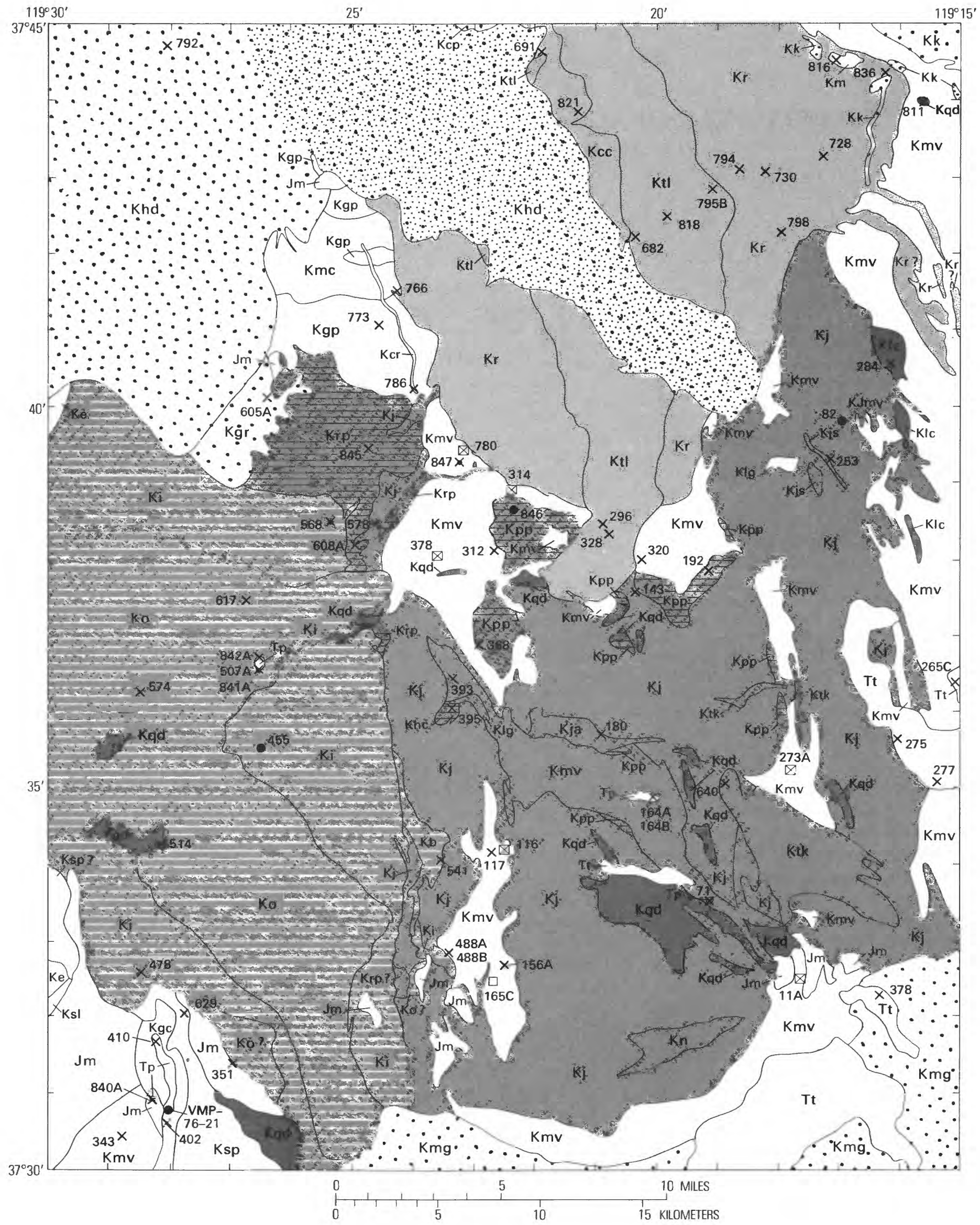




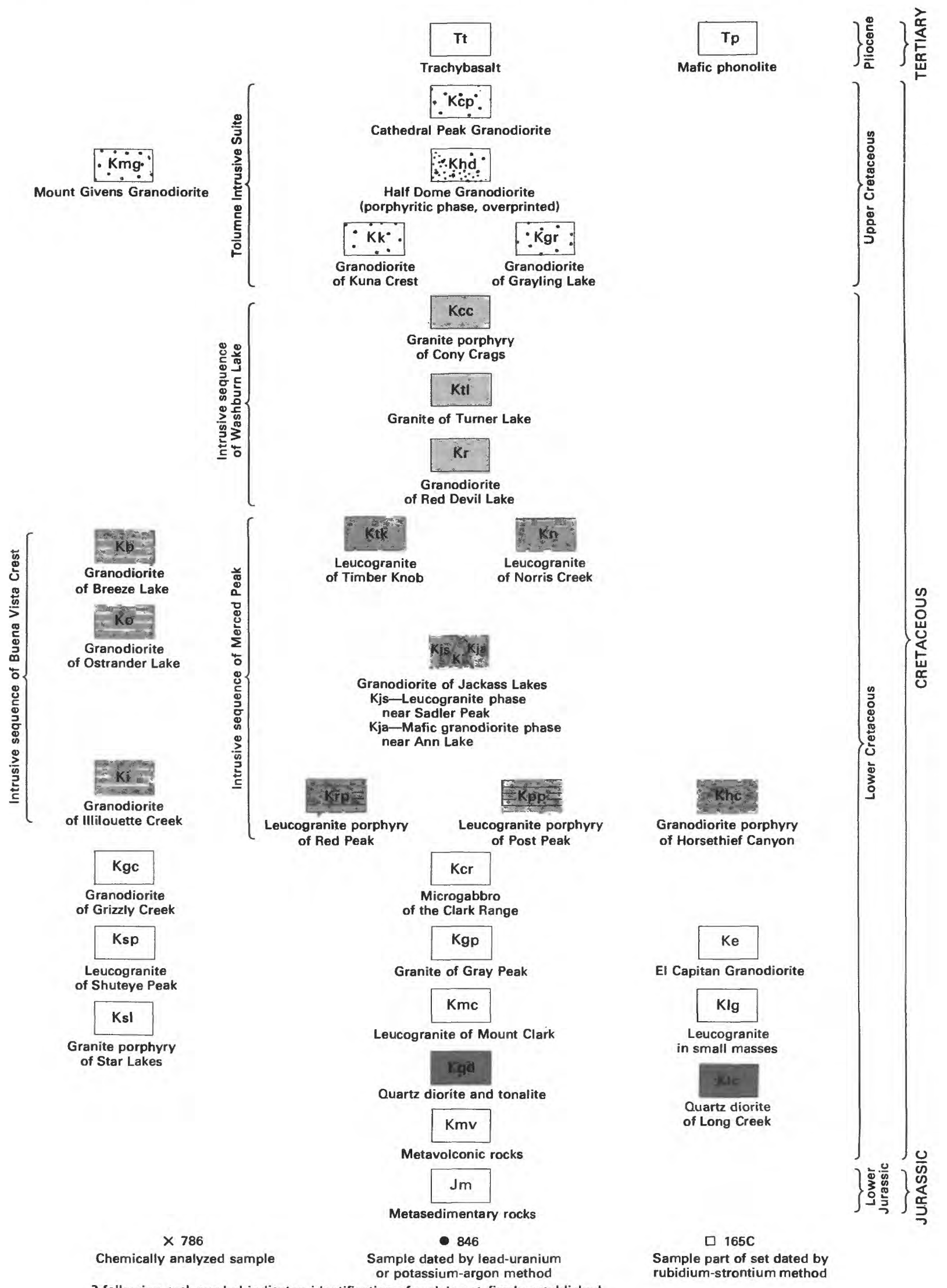

FIGURE 1. - Simplified bedrock geologic map of the Merced Peak quadrangle showing the locations of chemically analyzed and isotopically dated rock samples. Numbers refer to sample numbers in tables $\mathbf{1}$ through 5 . 
relative with the fossil-bearing metasedimentary rocks of the Boyden Cave pendant described by Jones and Moore (1973). Far more abundant are metavolcanic rocks, mostly blastoporphyritic, quartzo-feldspathic hornfels, epidote-amphibolite hornfels, and weakly foliated schist. They are predominantly derived from massive pyroclastic rocks of rhyodacitic composition but also include less abundant andesite, dacite, and rhyolite and sparse flows and shallow intrusions. The metavolcanic rocks along the eastern boundary of the quadrangle form the western margin of the Ritter Range pendant, described by Huber and Rinehart (1965). The metavolcanic rocks intrude metasedimentary rocks near the Strawberry Mine (Nokleberg, 1970; 1981) and are in turn intruded by many of the plutonic units, including quartz diorite and the intrusive sequence of Merced Peak. The rocks have yielded Early Cretaceous ages of 95 and 100 m.y. by rubidiumstrontium and lead-uranium methods, respectively (tables 1 and 2), approximately the same ages as those obtained from the granodiorite of Jackass Lakes. The similarity in age of these units and their close spatial association and similar chemical compositions suggest that they may well be genetically related. The geographic distribution of the metavolcanic rocks (excluding those in the southwest corner of the quadrangle) and the plutonic rocks of the Merced Peak intrusive sequence indicates that they may represent the preserved lower part of a caldera.

Plutonic rocks ranging from quartz diorite to leucogranite underlie most of the quadrangle. The rocks constitute many different plutons (29 units are distinguished in fig. 1) that are in sharp contact with one another. Most of the plutonic rocks are medium-grained granodiorite and granite, but they range from quartz diorite to leucogranite and from very coarsely porphyritic granodiorite to fine-grained aplite and leucogranite porphyry. Most of the area is underlain by plutonic rocks that are part of areally extensive plutons or suites of closely related plutons covering 100 to 1,000 $\mathrm{km}^{2}$. The intrusive sequences of Merced Peak and Washburn Lake are entirely or mostly confined to the quadrangle, but others extend · for many kilometers beyond the boundaries, particularly the Shuteye Peak,
TABLE 1.-Rubidium and strontium determinations on metavolcanic rocks ${ }^{12}$

[Rb and Sr determinations of MP-165B, MP-314, and MP-320 by isotope dilution, Carl Hedge analyst, U.S. Geological Survey, Denver, Colo.; all others by XRF, W. P. Doering analyst, U.S. Geological Survey, Denver, Colo. Strontium isotope analyses by R. Hildreth, U.S. Geological Survey, Denver, Colo., and R. Kistler, U.S. Geological Survey, Menlo Park, Calif. $\mathrm{Rb} / \mathrm{Sr}$ about \pm 3.0 percent or \pm 2.0 percent on those specimens determined by XRF or isotope dilution, respectively. Strontium isotope ratios are from \pm 0.02 percent to \pm 0.10 percent, normalized to ${ }^{a 6} \mathrm{Sr}^{\mathrm{B}} \mathrm{Sr}=0.1194$, and adjusted to $\mathrm{E}$ and $\mathrm{A} \mathrm{SrCO}$ ${ }^{7} \mathrm{Sr} /{ }^{8} \mathrm{Sr}=0.7080$. Isochron is regressed by the method of York (1966). Specimen MP-320 not used in isochron. $\lambda^{\mathrm{s} 7} \mathrm{Rb}=1.42 \times 10^{-11} / \mathrm{yr}$. Determinations $\mathrm{GC}-10$ to -204 from Warren Nokelberg, 1981; samples from the Strawberry Mine area]

\begin{tabular}{ccccc}
\hline Sample no. & $\mathrm{Rb}(\mathrm{ppm})$ & $\mathrm{Sr}(\mathrm{ppm})$ & $\mathrm{Rb} / \mathrm{Sr}$ & ${ }^{{ }^{7} \mathrm{Sr} /{ }^{\circ} \mathrm{Sr}}$ \\
\hline MP-11A -- & 85.8 & 551 & 0.156 & $0.7082 \pm .00014$ \\
MP-116 & 94.3 & 459 & .205 & $.7075 \pm .0007$ \\
MP-165B -- & 274 & 39.1 & 7.001 & $.7341 \pm .0002$ \\
MP-273A -- & 137 & 60.4 & 2.266 & $.7161 \pm .00014$ \\
MP-314-- & 44.1 & 472 & .093 & $.7072 \pm .0002$ \\
MP-320 -- & 159 & 47.3 & 3.362 & $.7149 \pm .0003$ \\
MP-378 -- & 130 & 233 & .556 & $.7088 \pm .00014$ \\
MP-780-- & 114 & 388 & .294 & $.7077 \pm .00014$ \\
GC-10--- & 59.0 & 504 & .117 & $.7073 \pm .00014$ \\
GC-41_--- & 122 & 387 & .315 & $.7073 \pm .00014$ \\
GC-50_-- & 115 & 366 & .314 & $.7077 \pm .00014$ \\
GC-62 & 189 & 96.9 & 1.947 & $.7142 \pm .00014$ \\
GC-204_-- & 136 & 210 & .646 & $.7096 \pm .00014$ \\
\hline
\end{tabular}

${ }^{1}$ Age $=94.7 \pm 4.6$ m.y.

${ }^{2}$ Initial ${ }^{87} \mathrm{Sr} /{ }^{38} \mathrm{Sr}=0.7068 \pm .0002$.

Mount Givens, and El Capitan plutons, the intrusive sequence of Buena Vista Crest, and the Tuolumne Intrusive Suite. Individual plutonic suites and large plutons typically range from hornblende granodiorite (or quartz diorite) rich in discoid mafic inclusions at the margins to fine-grained biotite granite or leucogranite porphyry at the core.

The plutonic rocks range in age from Early to Late Cretaceous (105 to $85 \mathrm{~m} . \mathrm{y}$.$) on the basis of radiometric$ ages from the Merced Peak quadrangle (listed in tables 2 and 3) and radiometric ages from adjacent areas. Most of the potassium-argon ages have been reduced by nearby younger intrusions, but the age of $95 \mathrm{~m} . \mathrm{y}$. for the hornblende from a sample of the granodiorite of Jackass Lakes (MP-82, table 3) is in good agreement with the age of $98 \mathrm{~m} . \mathrm{y}$. determined by lead-uranium methods on zircon from another sample of the unit (MP-520, table 2). That age and those determined on samples of the granodiorite of Red Devil Lake (MP-789, table 3) and of the metavolcanic rocks are consistent with the observed geologic relations and radiometric ages from adjacent areas, but the discordant age of 93 m.y. determined from a sample of the leucogranite porphyry of Post Peak appears to be too low by 6 m.y.

TABLE 2.-Uranium-lead age determinations on zircon from plutonic and metavolcanic rocks

[Determinations by T. W. Stern, U.S. Geological Survey, Reston, Va. Constants used: ${ }^{20} \mathrm{U} / 2 \mathrm{~s} \mathrm{U}$ 137.88; $238 \lambda=0.155 \times 10^{-7} \mathrm{yr}^{-1}$. Analytic uncertainties approximately \pm 3 percent. Age determined from $\left.{ }^{200} \mathrm{~Pb} /{ }^{238} \mathrm{U}\right]$

\begin{tabular}{|c|c|c|c|c|c|c|c|c|c|}
\hline Sample no. & Rock unit & $\begin{array}{c}\mathrm{Pb} \\
(\mathrm{ppm})\end{array}$ & $\underset{(\mathrm{ppm})}{\mathrm{U}}$ & $\begin{array}{c}\text { Th } \\
(\mathrm{ppm})\end{array}$ & $\begin{array}{c}{ }^{204} \mathrm{~Pb} \\
\text { (atomic } \\
\text { percent) }\end{array}$ & $\begin{array}{c}{ }^{286} \mathrm{~Pb} \\
\text { (atomic } \\
\text { percent) }\end{array}$ & $\begin{array}{c}{ }^{207} \mathrm{~Pb} \\
\text { (atomic } \\
\text { percent) }\end{array}$ & $\begin{array}{c}{ }^{200} \mathrm{~Pb} \\
\text { (atomic } \\
\text { percent) }\end{array}$ & $\begin{array}{l}\text { Age } \\
\text { (m.y.) }\end{array}$ \\
\hline MP-520 _- & Granodiorite of Jackass Lakes & 20.24 & 1140.4 & 951.8 & 0.03148 & 74.95 & 3.929 & 21.09 & 98 \\
\hline MP-568 & Granodiorite of Illilouette Creek & 19.64 & 1092.8 & 855.6 & .04319 & 75.78 & 4.081 & 20.10 & 100 \\
\hline MP-789 _ _ & Granodiorite of Red Devil Lake & 20.15 & 807.5 & 1948.9 & .02757 & 53.28 & 2.921 & 43.77 & 98 \\
\hline MP-846 & $\begin{array}{l}\text { Leucogranite porphyry of Post } \\
\text { Peak }\end{array}$ & 16.08 & 974.1 & 392.4 & .2873 & 80.71 & 5.227 & 13.77 & 93 \\
\hline MP-847 _ _- & Metavolcanic rocks & 11.12 & 600.8 & 285.5 & .1877 & 76.00 & 6.103 & 17.71 & 100 \\
\hline
\end{tabular}


TABLE 3.-Potassium-argon age determinations on minerals from granitic and volcanic rocks

[Constants used ${ }^{40} \mathrm{~K}: \gamma \epsilon+\lambda_{\epsilon}=0.581 \times 10^{-10}$ year ${ }^{-1}, \gamma \beta=4.962 \times 10^{-10}$ year-1; isotopic abundance $1.167 \times 10^{-4}$ moles ${ }^{40} \mathrm{~K}$ per mole $\mathrm{K}$. Radiogenic argon $=\mathrm{r}^{\mathrm{T}} \mathrm{Ar}$; total argon $=\mathrm{t}^{\mathrm{0}} \mathrm{Ar}$. Analysts for MP-82, MP-455, and MP-789, R. W. Kistler and Lois Schlocker, U.S. Geological Survey, Menlo Park, Calif. Analyst for VMP-76-21 and MP-841A, Elliot Sims, under the direction of G. Brent Dalrymple, U.S. Geological Survey, Menlo Park, Calif.]

\begin{tabular}{|c|c|c|c|c|c|c|c|c|}
\hline \multicolumn{9}{|c|}{ Argon } \\
\hline Sample & Rock unit & Mineral & $\begin{array}{c}\mathrm{K}_{2} \mathrm{O} \\
\text { (weight } \\
\text { percent) }\end{array}$ & \multicolumn{3}{|c|}{$\begin{array}{c}{ }^{40} \mathrm{Ar}_{\text {rad }} \\
\left(\text { moles/g }^{2} / \mathrm{g}\right. \\
\left.\times 10^{11}\right)\end{array}$} & $\begin{array}{l}{ }^{40} \mathrm{Ar} \text { rad } \\
\text { (percent) }\end{array}$ & $\begin{array}{c}\text { Age } \\
\text { (m.y.) }\end{array}$ \\
\hline \multirow[t]{2}{*}{$\mathrm{MP}-82$} & \multirow{2}{*}{ Granodiorite of Jackass Lakes } & Biotite & 9.21 & \multicolumn{3}{|c|}{115.83} & 81 & $85.5 \pm 2.1$ \\
\hline & & Hornblende & 1.17 & \multicolumn{3}{|c|}{16.25} & 71 & $95.2 \pm 2.8$ \\
\hline \multirow{2}{*}{$\mathrm{MP}-455$} & \multirow{2}{*}{$\begin{array}{l}\text { Granodiorite of Illilouette } \\
\text { Creek }\end{array}$} & Biotite & 9.30 & \multicolumn{3}{|c|}{121.50} & 65 & $88.7 \pm 2.2$ \\
\hline & & Hornblende & 1.00 & \multirow{2}{*}{\multicolumn{3}{|c|}{$\begin{array}{r}12.53 \\
116.83\end{array}$}} & 63 & $85.1 \pm 2.5$ \\
\hline \multirow[t]{2}{*}{ Mp-789 _-_- } & Granodiorite of Red Devil & Biotite & 9.43 & & & & 79 & $84.2 \pm 2.1$ \\
\hline & Lake & Hornblende & .82 & \multicolumn{3}{|c|}{$\begin{array}{r}116.83 \\
10.57\end{array}$} & 38 & $87.4 \pm 2.6$ \\
\hline VMP-76-21 - & $\begin{array}{l}\text { Mafic phonolite near Grizzly } \\
\text { Creek }\end{array}$ & Phlogopite & 9.83 & \multicolumn{3}{|c|}{5.105} & 25.2 & $3.60 \pm 0.06$ \\
\hline \multirow[t]{2}{*}{ MP-841A --- } & $\begin{array}{l}\text { Mafic phonolite near Merced } \\
\text { Pass }\end{array}$ & Phlogopite & 8.80 & \multicolumn{3}{|c|}{3.859} & 32.6 & $3.04 \pm 0.04$ \\
\hline & & & ${ }^{40} \mathrm{Ar} /{ }^{39} \mathrm{Ar}$ & ${ }^{37} \mathrm{Ar} /{ }^{39} \mathrm{Ar}{ }^{1}$ & ${ }^{36} \mathrm{Ar} /{ }^{39} \mathrm{Ar}$ & $J^{2}$ & & \\
\hline VMP-76-21 & $\begin{array}{l}\text { Mafic phonolite near Grizzly } \\
\text { Creek }\end{array}$ & Phlogopite & 1.949 & 0.02496 & 0.00541 & 0.005678 & 17.8 & $3.55 \pm 0.21$ \\
\hline MP-841A -- & $\begin{array}{l}\text { Mafic phonolite near Merced } \\
\text { Pass }\end{array}$ & Phlogopite $^{3}$ & 2.091 & .03117 & .00573 & .005702 & 18.8 & $4.05 \pm 0.22$ \\
\hline $\begin{array}{l}\text { MP-841A } \\
\text { MP_841A }\end{array}$ & - & Phlogopite ${ }^{4}$ & 1.729 & .03760 & .00463 & .005702 & 20.7 & $3.68 \pm 0.18$ \\
\hline MP-841A _ _- & - & Sanidine & .8876 & .1674 & .00180 & .005702 & 39.5 & $3.60 \pm 0.10$ \\
\hline
\end{tabular}

${ }^{1}$ Corrected for ${ }^{37} \mathrm{Ar}$ decay $\left(\mathrm{t}_{1 / 2}=35.1\right.$ days).

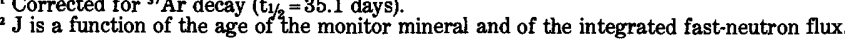

Volcanic rocks of Tertiary age occur near Jackass Meadow (near the southeast corner of the quadrangle), along Cora Creek (10 km farther north), and at several small isolated localities. Most are in flows and sparse cinder deposits of trachybasalt and less abundant trachyandesite similar to the Tertiary volcanic rocks of adjacent areas described by Hamilton and Neuerburg (1956). Small flows along Grizzly Creek and near Merced Pass and a flow and volcanic neck along the west fork of Granite Creek, however, represent a rare composition for volcanic rocks; they are phlogopite-bearing, ultrapotassic, mafic phonolite similar to the orendite and olivine orendite of the Leucite Hills, Wyoming (Carmichael, 1967), and the minette dikes of the Navajo region (Williams, 1936). The mineralogy, petrology, and geochemistry of these volcanic rocks have recently been described by Van Kooten (1980). Trachybasalt flows in adjacent quadrangles have yielded Pliocene potassiumargon ages of 2.9 to 3.6 m.y. (Dalrymple 1963, 1964). Similar ages of about 3.6 m.y. were determined by potassium-argon determination of phlogopite and sanidine from the Merced Pass and Grizzly Creek localities (as listed in table 3, samples MP-841A and VMP-76-21), indicating that the trachybasalt and phonolite are at least in part contemporaneous.

The Lower Cretaceous and older plutonic and metamorphic rocks, but not the Upper Cretaceous plutonic rocks, are cut by northwest-trending shears along several zones that cross the quadrangle. These shears are not indicated on the simplified geologic map (fig. 1) but are shown and discussed on the geologic map of the Merced Peak quadrangle (Peck, 1980). All the
3 Screened to $60-100$ mesh.
${ }^{4}$ Screened to $100-200$ mesh.

plutonic and metamorphic rocks are cut by steeply dipping regional joints, as shown in figure 2. This figure, which was prepared from aerial photographs of the quadrangle, shows a dominant north- to northnortheast-trending set of joint cracks crossed by a less prominent west- to northwest-trending set.

Glacial deposits, primarily of Wisconsinan Age, cover broad areas of the quadrangle, particularly along Granite, Illilouette, and Chiquito Creeks and the North and South Forks of the Merced River. These deposits are not shown on the simplified geologic map (fig. 1), but they are shown and described in the geologic map of the Merced Peak quadrangle (Peck, 1980).

\section{ANALYTIC DATA}

During the course of the geologic mapping, 845 rock samples were collected, including 520 of plutonic rocks. Of these, 258 were analyzed modally, and 41 were also analyzed chemically and spectrographically, as were 15 samples of metavolcanic rocks and 11 samples of Tertiary volcanic rocks. The locations of chemically and isotopically analyzed samples are plotted in figure 1 and listed in table 4 together with the chemical and spectrographic analyses, norms, and modes. Modes were determined by counting 1,000 to 2,000 regularly spaced points on rock slabs of not less than $40 \mathrm{~cm}^{2}$, on which potassium feldspar had been selectively stained yellow and plagioclase red (Norman, 1974). Four constituents were counted-quartz, potassium feldspar, plagioclase, and mafic minerals. The locations of the modally analyzed samples are plotted in figure 3 and listed in table 5 , along with modes and specific gravities. The percent- 


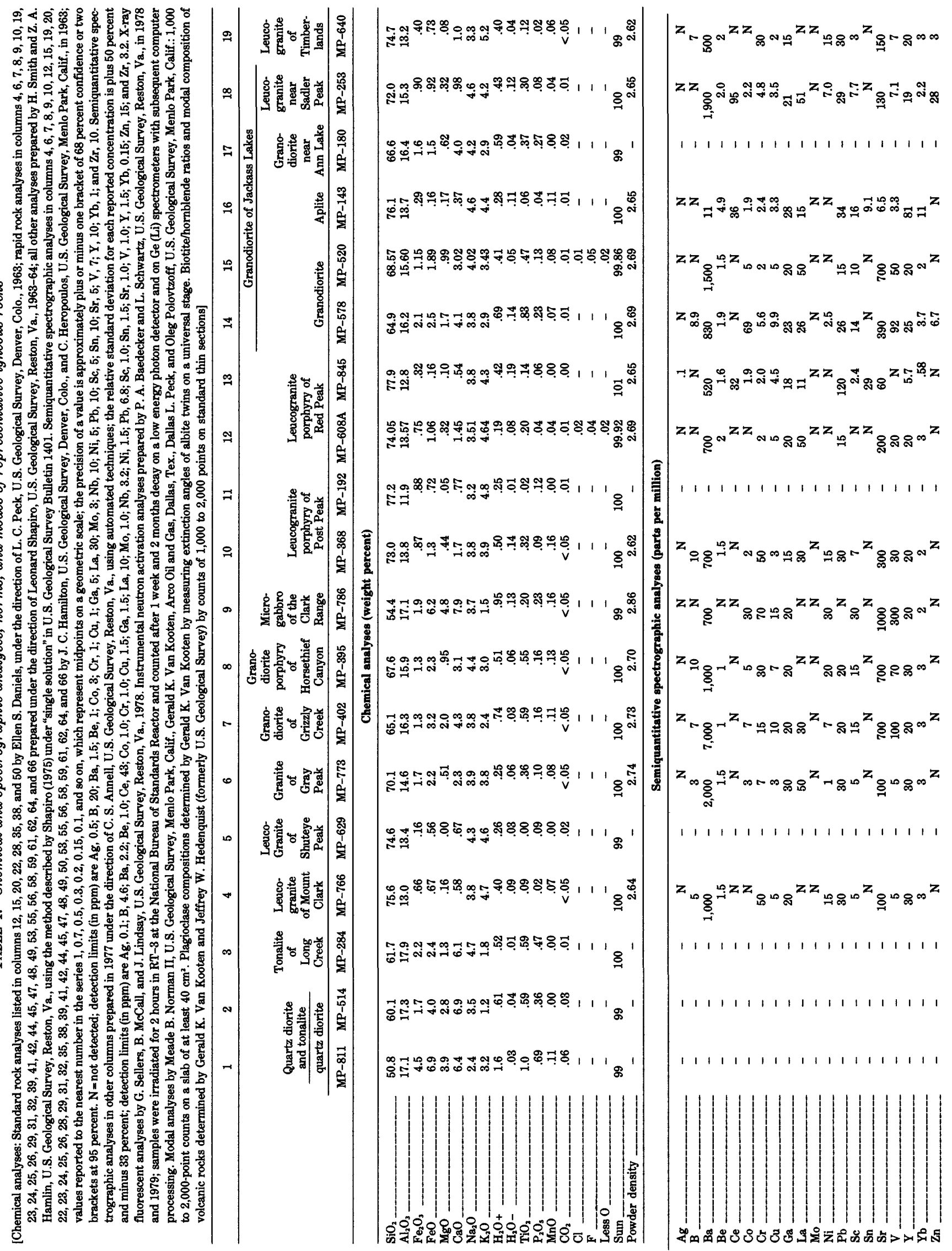


MERCED PEAK QUADRANGLE, CENTRAL SIERRA NEVADA, CALIFORNIA-ANALYTIC DATA

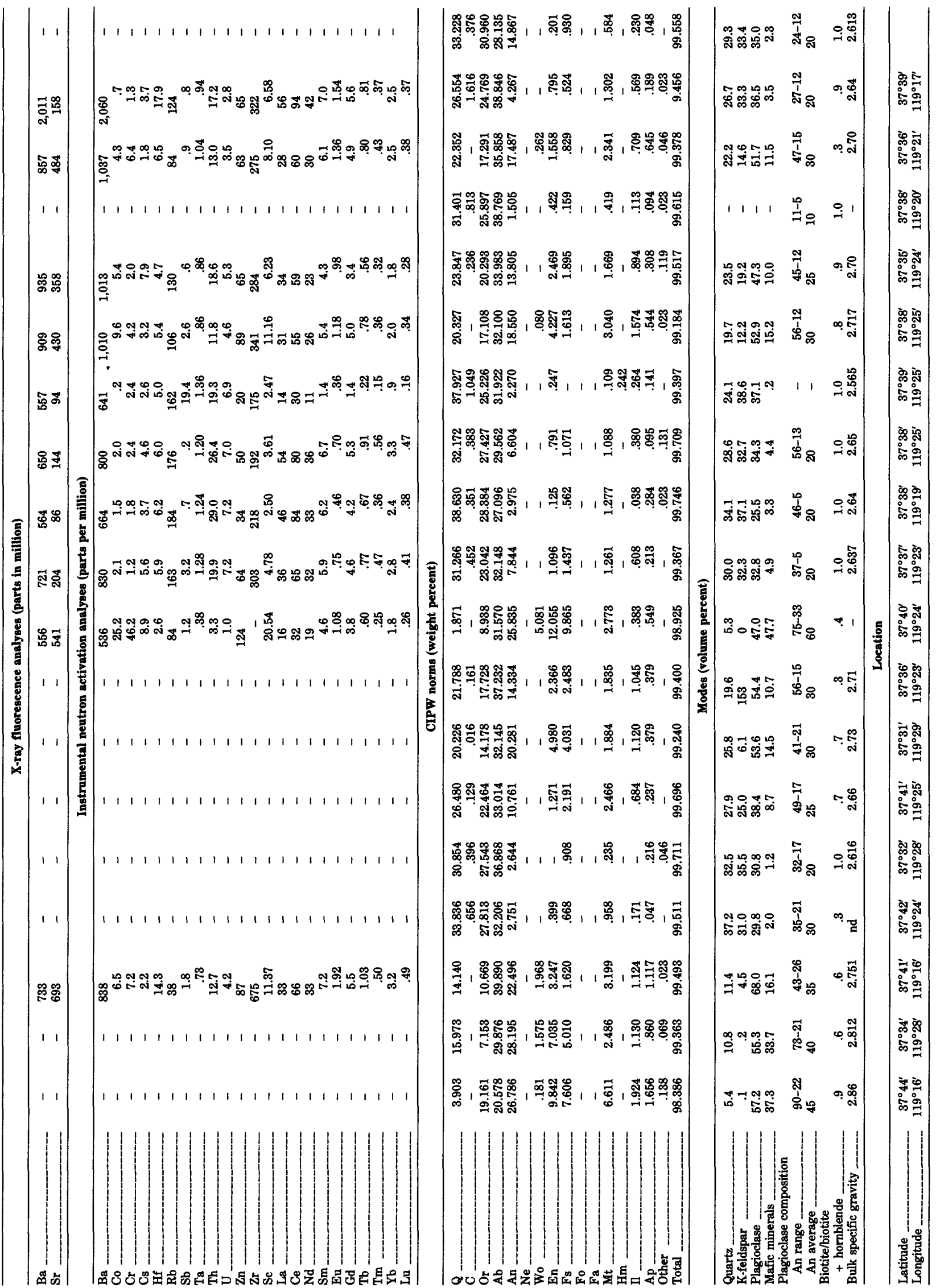


SHORTER CONTRIBUTIONS TO GENERAL GEOLOGY

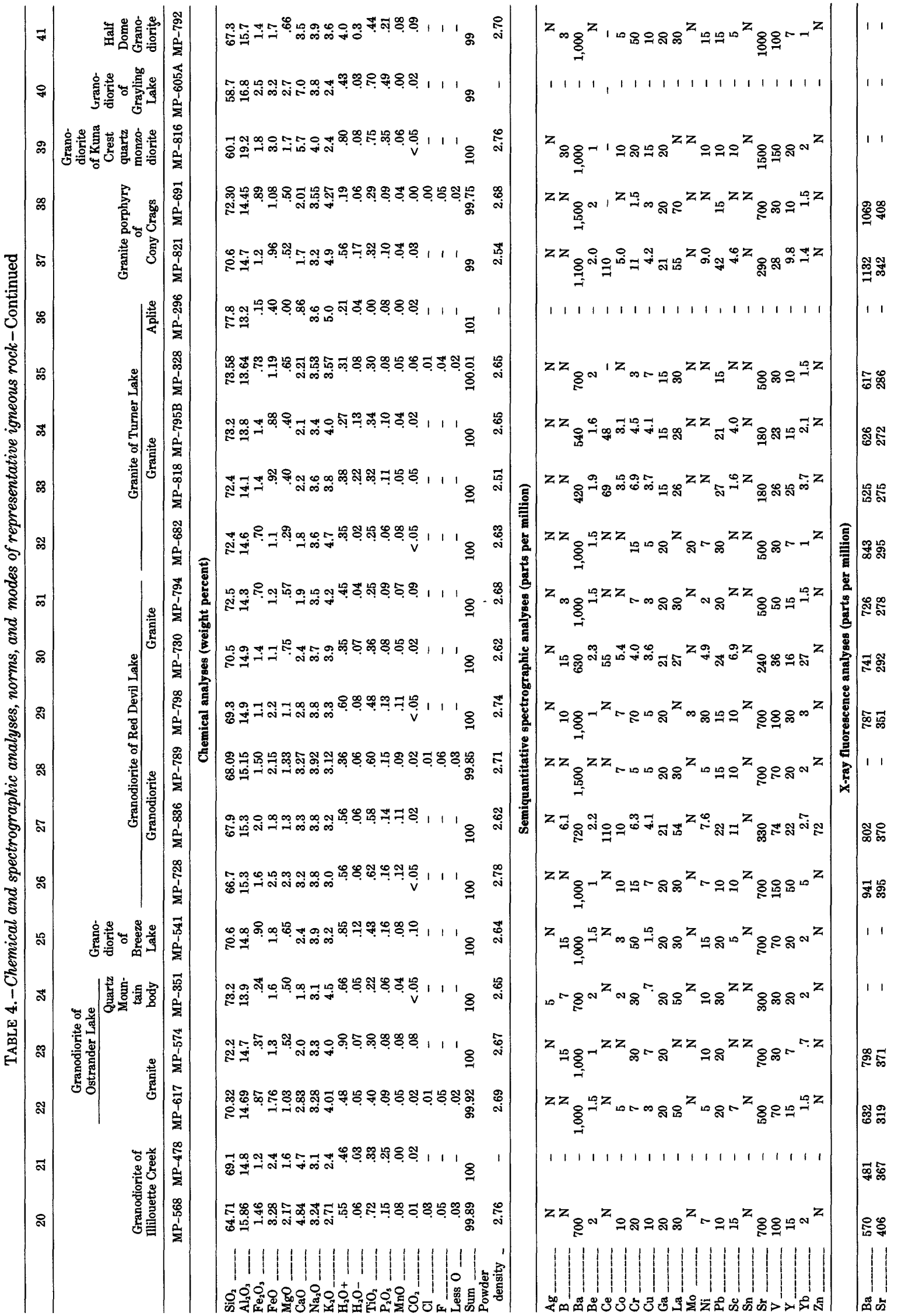


MERCED PEAK QUADRANGLE, CENTRAL SIERRA NEVADA, CALIFORNIA-ANALYTIC DATA

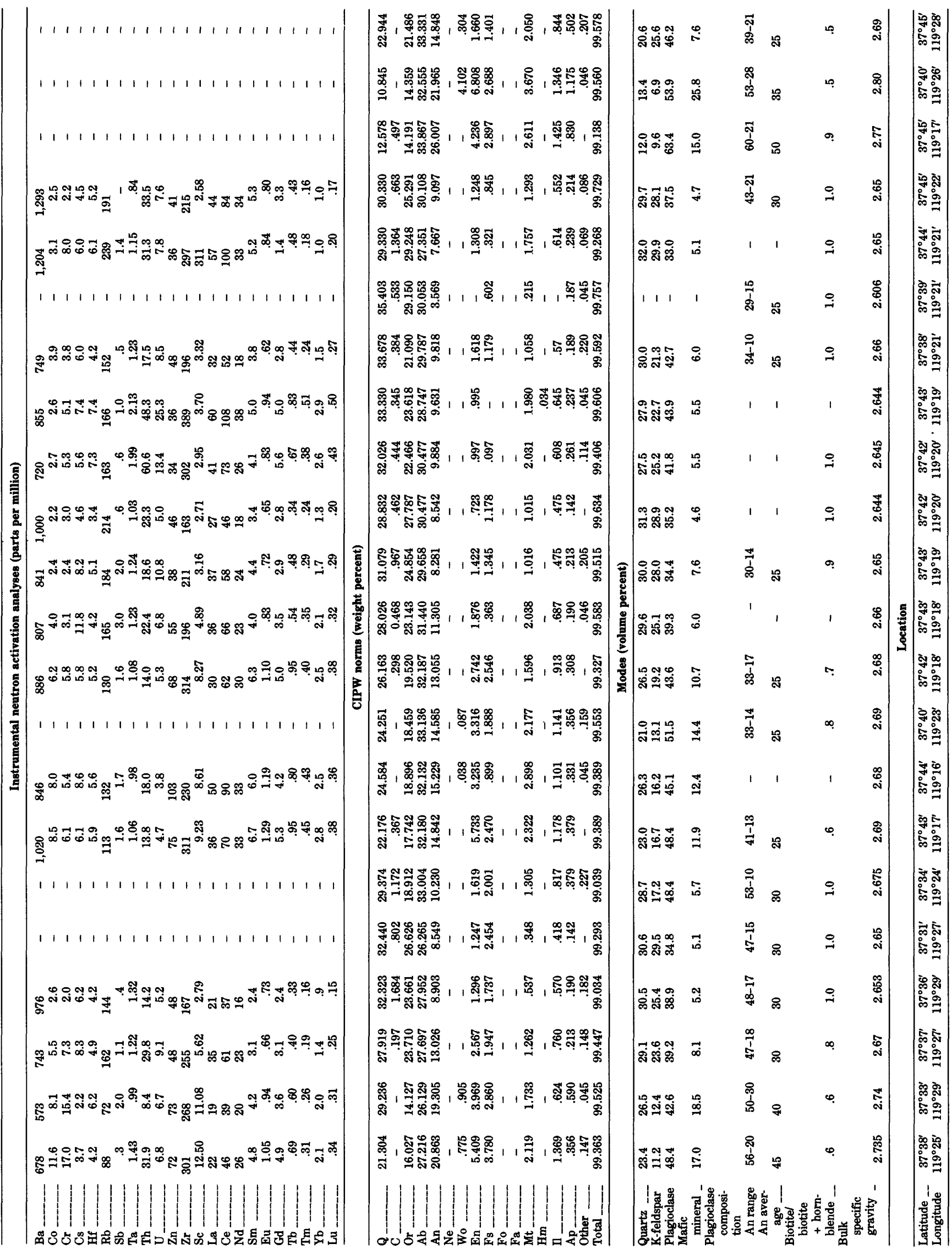




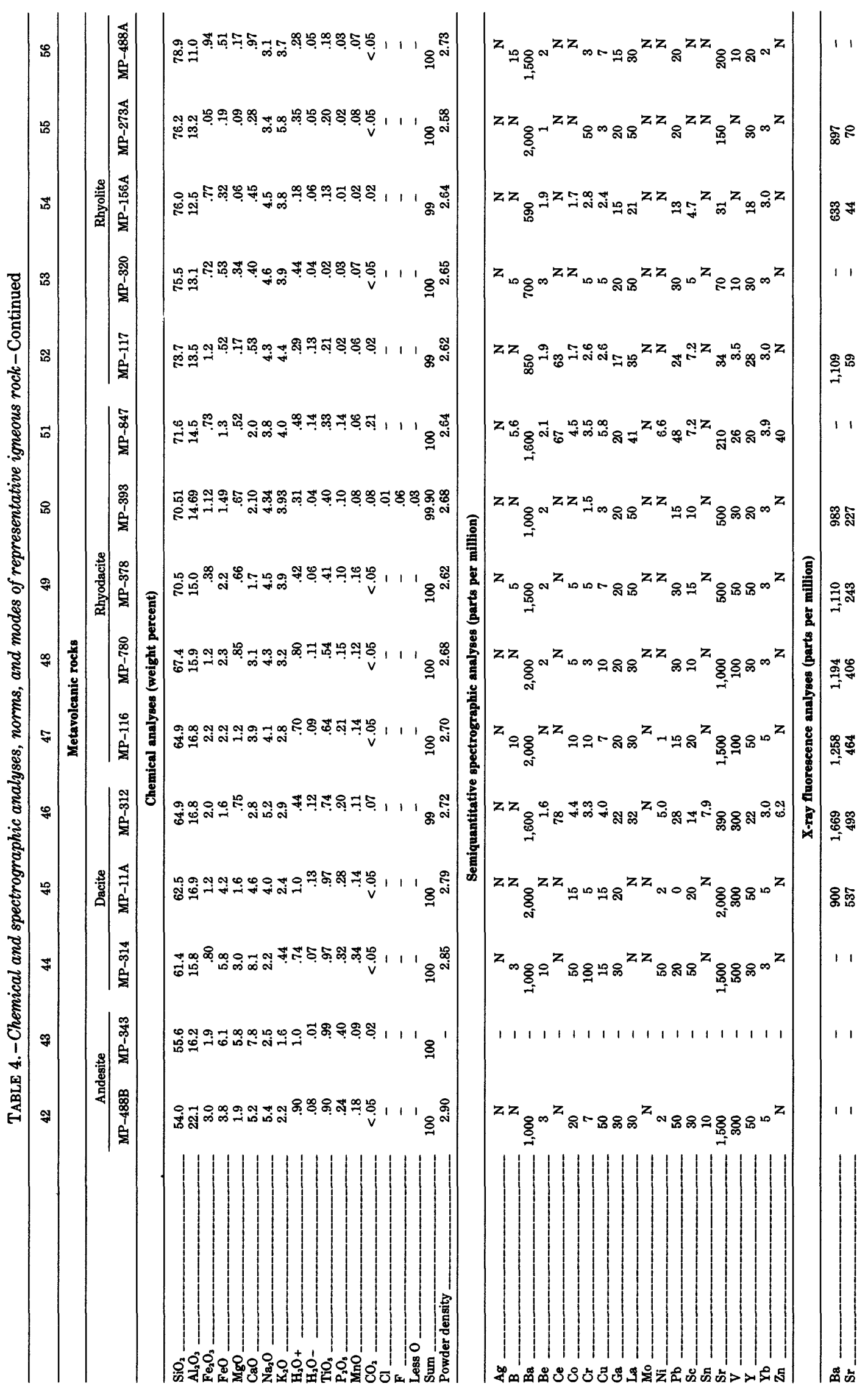


MERCED PEAK QUADRANGLE, CENTRAL SIERRA NEVADA, CALIFORNIA-ANALYTIC DATA

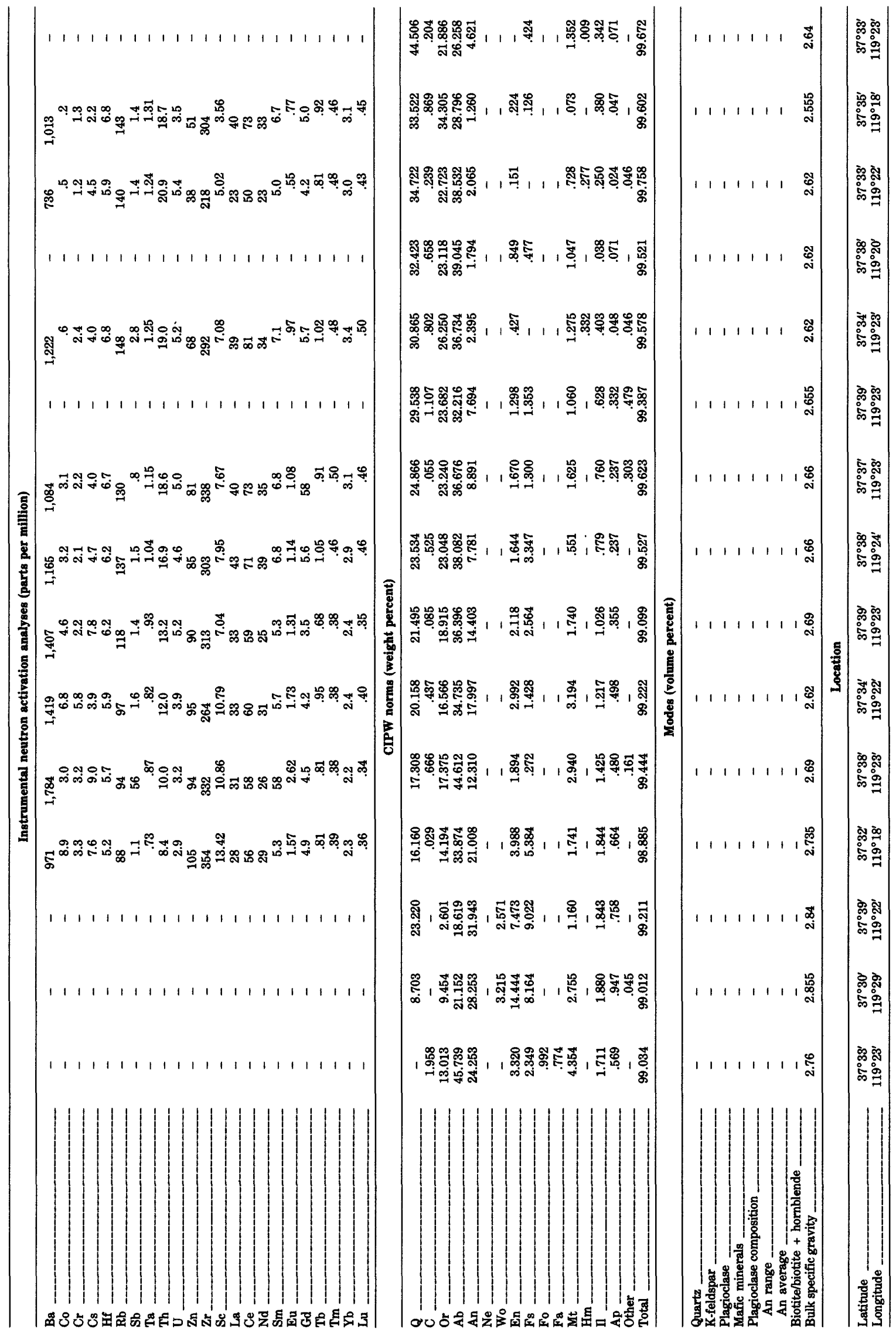




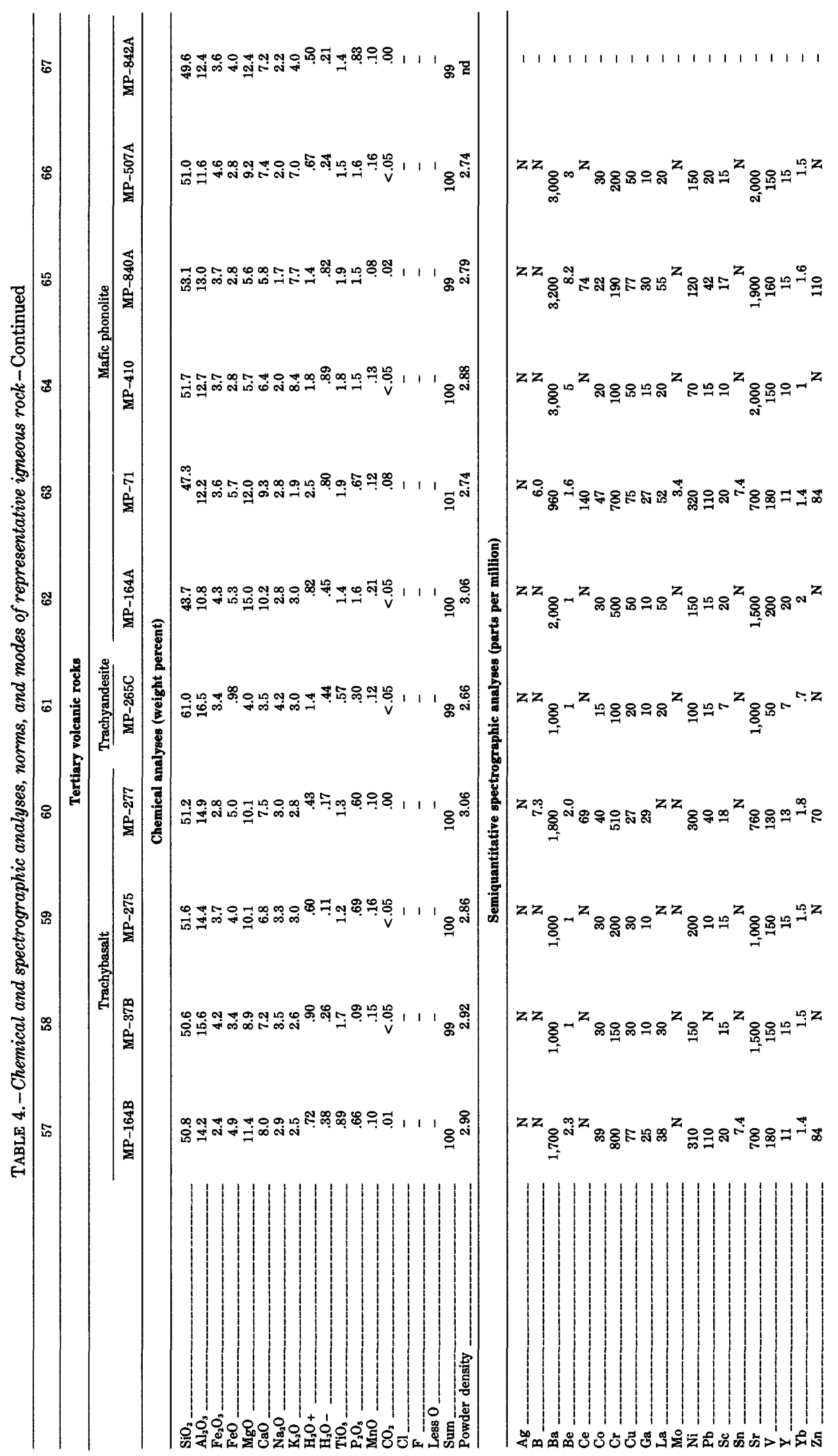


MERCED PEAK QUADRANGLE, CENTRAL SIERRA NEVADA, CALIFORNIA-ANALYTIC DATA

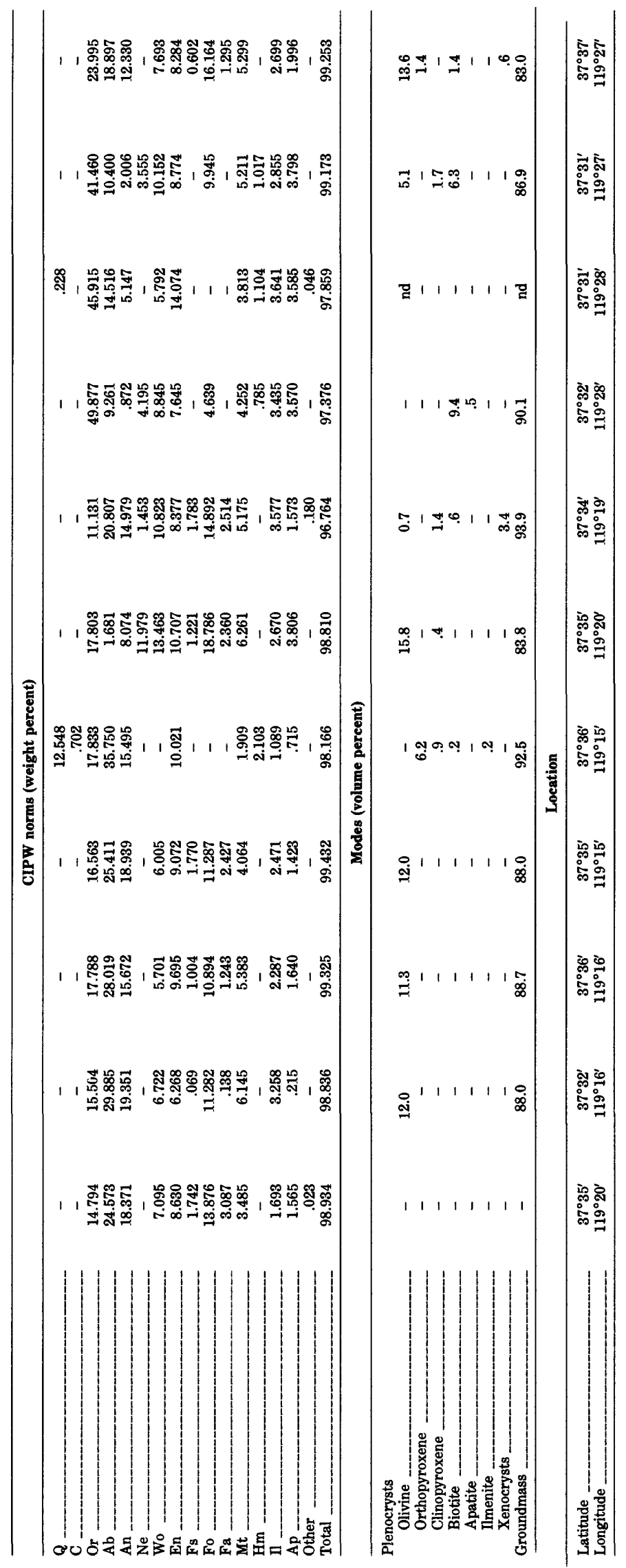




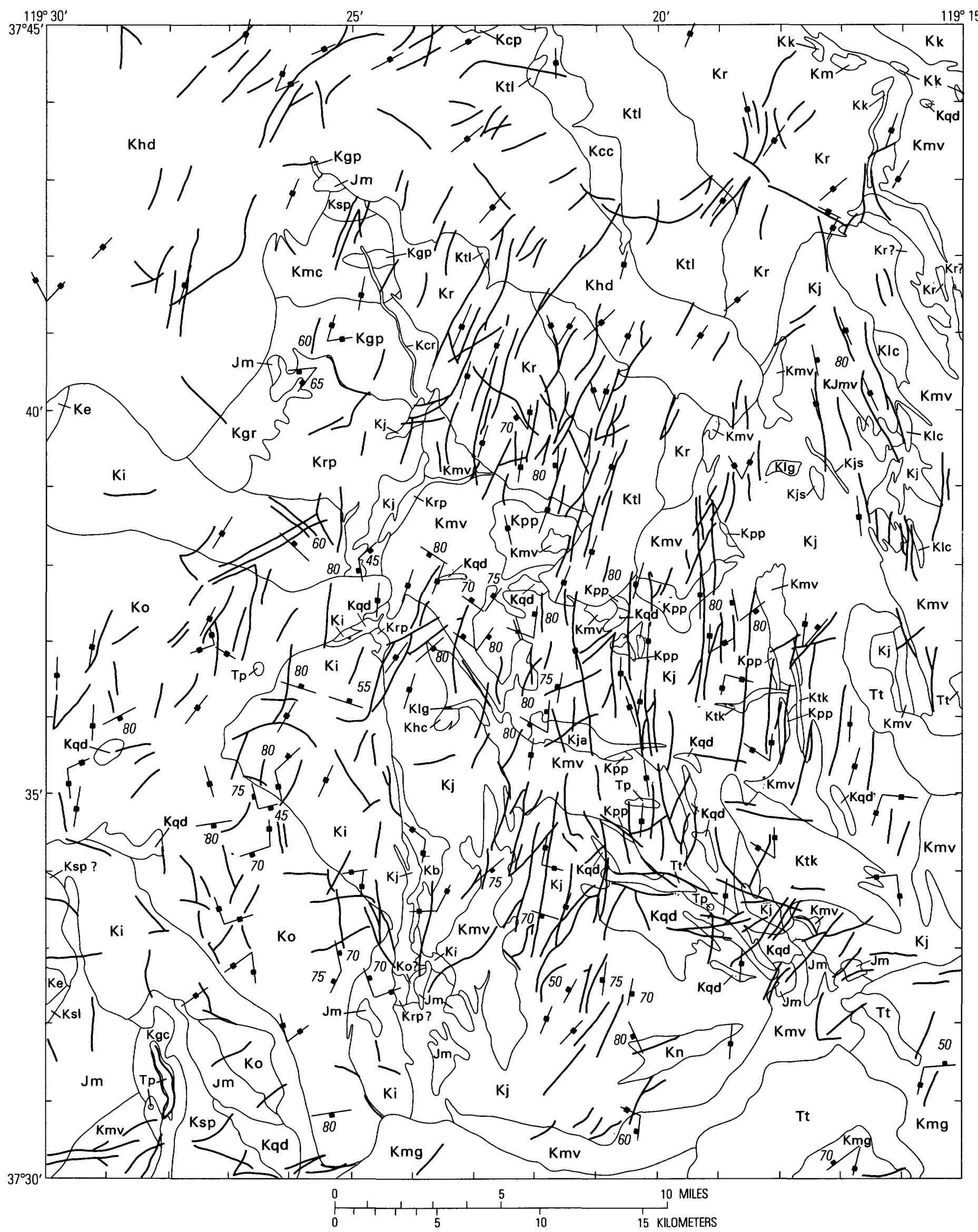

Figure 2.-Simplified bedrock geologic map of the Merced Peak quadrangle, showing steeply inclined regional joints (heavy lines) observed on aerial photographs and the attitude of joints as observed on the ground. See figure 1 for explanation. Figure prepared by J. W. Hedenquist. 
MERCED PEAK QUADRANGLE, CENTRAL SIERRA NEVADA, CALIFORNIA-ANALYTIC DATA

TABLE 5.-Modes, specific gravity, and location of plutonic rocks

\begin{tabular}{|c|c|c|c|c|c|c|c|c|}
\hline Sample no. & $\begin{array}{c}\text { Map symbol } \\
\text { for rock } \\
\text { unit }\end{array}$ & $\begin{array}{l}\text { Latitude } \\
37^{\circ} 30^{\prime}-45^{\prime}\end{array}$ & $\begin{array}{c}\text { Longitude } \\
119^{\circ} 15^{\prime}-30^{\prime}\end{array}$ & $\begin{array}{c}\begin{array}{c}\text { Plagioclase } \\
\text { (volume percent) }\end{array} \\
\text { volut }\end{array}$ & $\begin{array}{c}\text { Alkali } \\
\text { feldspar } \\
\text { (volume percent) }\end{array}$ & $\begin{array}{c}\text { Quartz } \\
\text { (volume percent) }\end{array}$ & $\begin{array}{c}\text { Mafic } \\
\text { minerals } \\
\text { (volume percent) }\end{array}$ & $\begin{array}{l}\text { Specific } \\
\text { gravity } \\
\left(\mathrm{g} / \mathrm{cm}^{3}\right)\end{array}$ \\
\hline MP-1 & $\mathrm{Kmg}$ & $37^{\circ} 32^{\prime}$ & $119^{\circ} 16^{\prime}$ & 48.8 & 9.9 & 13.0 & 23.3 & 2.751 \\
\hline MP-4A & $\mathrm{Kmg}$ & $37^{\circ} 32^{\prime}$ & $119^{\circ} 16^{\prime}$ & 51.5 & 16.4 & 20.4 & 11.7 & 2.688 \\
\hline MP-19 & $\mathbf{K j}$ & $37^{\circ} 31^{\prime}$ & $119^{\circ} 19^{\prime}$ & 43.2 & 18.6 & 29.6 & 8.7 & 2.675 \\
\hline MP-39B & $\mathbf{K} \mathbf{j}$ & $37^{\circ} 33^{\prime}$ & $119^{\circ} 17^{\prime}$ & 55.9 & 3.9 & 29.4 & 10.8 & 2.71 \\
\hline MP-56 & $\mathbf{K j}$ & $37^{\circ} 34^{\prime}$ & $119^{\circ} 16^{\prime}$ & 61.8 & 4.6 & 18.6 & 14.9 & 2.715 \\
\hline MP-61 & $\mathbf{K j}$ & $37^{\circ} 33^{\prime}$ & $119^{\circ} 19^{\prime}$ & 49.4 & 16.2 & 25.7 & 8.7 & 2.675 \\
\hline MP-65 & $\mathbf{K} \mathbf{j}$ & $37^{\circ} 32^{\prime}$ & $119^{\circ} 21^{\prime}$ & 50.1 & 20.4 & 21.9 & 7.6 & 2.64 \\
\hline MP-68E & $\mathrm{Kmg}$ & $37^{\circ} 30^{\prime}$ & $119^{\circ} 21^{\prime}$ & 52.4 & 15.1 & 23.3 & 9.2 & 2.702 \\
\hline MP-77 & $\mathbf{K} \mathbf{j}$ & $37^{\circ} 40^{\prime}$ & $119^{\circ} 16^{\prime}$ & 44.9 & 20.7 & 24.3 & 10.1 & 2.68 \\
\hline MP-82 & $\mathbf{K} \mathbf{j}$ & $37^{\circ} 40^{\prime}$ & $119^{\circ} 17^{\prime}$ & 58.1 & 11.2 & 19.4 & 11.3 & 2.69 \\
\hline MP-89B & $\mathbf{K j}$ & $37^{\circ} 39^{\prime}$ & $119^{\circ} 16^{\prime}$ & 43.1 & 23.9 & 24.0 & 9.0 & 2.64 \\
\hline MP-93 & $\mathbf{K j}$ & $37^{\circ} 38^{\prime}$ & $119^{\circ} 16^{\prime}$ & 51.7 & 19.4 & 19.5 & 9.4 & 2.68 \\
\hline MP-97 & Ktk & $37^{\circ} 34^{\prime}$ & $119^{\circ} 18^{\prime}$ & 40.5 & 23.0 & 31.3 & 5.2 & 2.63 \\
\hline MP-99B & $\mathbf{K} \mathbf{j}$ & $37^{\circ} 35^{\prime}$ & $119^{\circ} 18^{\prime}$ & 43.2 & 24.8 & 22.5 & 9.5 & 2.67 \\
\hline MP-124B & Kqd & $37^{\circ} 33^{\prime}$ & $119^{\circ} 21^{\prime}$ & 54.0 & 2.3 & 18.8 & 24.8 & 2.785 \\
\hline MP-133 & $\mathrm{Kpp}$ & $37^{\circ} 38^{\prime}$ & $119^{\circ} 20^{\prime}$ & 21.7 & 44.1 & 28.7 & 5.5 & 2.623 \\
\hline MP-134B & $\mathrm{Ktl}$ & $37^{\circ} 38^{\prime}$ & $119^{\circ} 21^{\prime}$ & 40.3 & 22.5 & 29.6 & 7.6 & 2.66 \\
\hline MP-135B & Ktl & $37^{\circ} 38^{\prime}$ & $119^{\circ} 22^{\prime}$ & 38.7 & 21.7 & 31.2 & 8.4 & 2.65 \\
\hline MP-138B & Kpp? & $37^{\circ} 37^{\prime}$ & $119^{\circ} 23^{\prime}$ & 36.1 & 31.5 & 26.8 & 5.6 & 2.625 \\
\hline MP-143 & $\mathbf{K j}$ & $37^{\circ} 38$ & $119^{\circ} 20^{\prime}$ & & & & & \\
\hline MP-154A & $\mathbf{K j}$ & $37^{\circ} 34^{\prime}$ & $119^{\circ} 22^{\prime}$ & 50.6 & 14.8 & 21.4 & 13.1 & 2.71 \\
\hline MP-155 & $\mathbf{K j}$ & $37^{\circ} 33^{\prime}$ & $119^{\circ} 22^{\prime}$ & 31.7 & 33.6 & 31.5 & 3.2 & 2.635 \\
\hline MP-158 & $\mathbf{K j}$ & $37^{\circ} 34^{\prime}$ & $119^{\circ} 22^{\prime}$ & 46.8 & 17.4 & 25.5 & 10.2 & 2.682 \\
\hline MP-169C & $\mathbf{K j}$ & $37^{\circ} 36^{\prime}$ & $119^{\circ} 18^{\prime}$ & 52.0 & 17.9 & 22.6 & 7.5 & 2.662 \\
\hline MP-175 & $\mathbf{K j}$ & $37^{\circ} 37^{\prime}$ & $119^{\circ} 21^{\prime}$ & 48.8 & 19.8 & 23.0 & 8.4 & 2.663 \\
\hline MP-180 & Kja & $37^{\circ} 36^{\prime}$ & $119^{\circ} 21^{\prime}$ & 51.7 & 14.6 & 22.2 & 11.5 & 2.70 \\
\hline MP-182 & Kja & $37^{\circ} 36^{\prime}$ & $119^{\circ} 22^{\prime}$ & 48.0 & 17.9 & 23.9 & 10.2 & 2.687 \\
\hline MP-183 & $\mathbf{K j a}$ & $37^{\circ} 36^{\prime}$ & $119^{\circ} 22^{\prime}$ & 47.1 & 13.8 & 31.9 & 7.2 & 2.67 \\
\hline MP-185A & $\mathbf{K j a}$ & $37^{\circ} 36^{\prime}$ & $119^{\circ} 20^{\prime}$ & 54.9 & 13.9 & 19.3 & 11.9 & 2.712 \\
\hline MP-187 & Kmg & $37^{\circ} 30^{\prime}$ & $119^{\circ} 25^{\prime}$ & 44.3 & 23.5 & 20.6 & 11.6 & 2.677 \\
\hline MP-188 & $\mathbf{K j}$ & $37^{\circ} 39^{\prime}$ & $119^{\circ} 18^{\prime}$ & 61.6 & 7.2 & 16.0 & 15.2 & 2.73 \\
\hline MP-192 -- & Kpp & $37^{\circ} 38^{\prime}$ & $119^{\circ} 19^{\prime}$ & 25.5 & 37.1 & 34.1 & 3.3 & 2.64 \\
\hline MP-195 & $\mathbf{K j}$ & $37^{\circ} 37^{\prime}$ & $119^{\circ} 19^{\prime}$ & 45.4 & 22.0 & 23.8 & 8.8 & 2.665 \\
\hline MP-197 _- & $\mathbf{K j}$ & $37^{\circ} 39^{\prime}$ & $119^{\circ} 18^{\prime}$ & 43.1 & 25.2 & 26.2 & 5.5 & 2.65 \\
\hline MP-198 & Klg & $37^{\circ} 39^{\prime}$ & $119^{\circ} 18^{\prime}$ & 31.0 & 30.3 & 34.6 & 4.1 & 2.626 \\
\hline MP-217 & $\mathrm{Kpp}$ & $37^{\circ} 38^{\prime}$ & $119^{\circ} 22^{\prime}$ & 36.4 & 27.9 & 28.2 & 7.5 & 2.655 \\
\hline MP-221 & $\mathbf{K r}$ & $37^{\circ} 40^{\prime}$ & $119^{\circ} 22^{\prime}$ & 45.9 & 19.9 & 24.1 & 10.1 & 2.68 \\
\hline MP-253 & $\mathrm{Kjs}$ & $37^{\circ} 39^{\prime}$ & $119^{\circ} 17^{\prime}$ & 36.5 & 33.3 & 26.7 & 3.5 & 2.64 \\
\hline MP-254 & Kjs & $37^{\circ} 39^{\prime}$ & $119^{\circ} 17^{\prime}$ & 41.7 & 24.3 & 25.7 & 8.3 & 2.659 \\
\hline MP-257A & $\mathbf{K j}$ & $37^{\circ} 38^{\prime}$ & $119^{\circ} 17^{\prime}$ & 47.9 & 16.4 & 25.1 & 10.5 & 2.66 \\
\hline MP-262 & Ktk & $37^{\circ} 36^{\prime}$ & $119^{\circ} 18^{\prime}$ & 40.0 & 24.0 & 31.4 & 4.6 & 2.67 \\
\hline MP-263 & Ktk & $37^{\circ} 37^{\prime}$ & $119^{\circ} 18^{\prime}$ & 29.3 & 32.2 & 33.9 & 4.6 & 2.62 \\
\hline MP-271 & $\mathbf{K} \mathbf{j}$ & $37^{\circ} 35^{\prime}$ & $119^{\circ} 17^{\prime}$ & 52.2 & 15.3 & 15.8 & 16.7 & 2.73 \\
\hline $\mathrm{MP}-274 \mathrm{~B}$ & Kpp & $37^{\circ} 36^{\prime}$ & $119^{\circ} 18^{\prime}$ & 30.4 & 30.8 & 35.4 & 3.4 & 2.64 \\
\hline MP-284 & Klc & $37^{\circ} 41^{\prime}$ & $119^{\circ} 16^{\prime}$ & 68.0 & 4.5 & 11.4 & 16.1 & 2.751 \\
\hline MP-285 & $\mathbf{K} \mathbf{j}$ & $37^{\circ} 41^{\prime}$ & $119^{\circ} 17^{\prime}$ & 44.6 & 21.5 & 26.2 & 7.7 & 2.677 \\
\hline MP-289 & Kr? & $37^{\circ} 42^{\prime}$ & $119^{\circ} 16^{\prime}$ & 62.4 & 7.5 & 15.8 & 14.3 & 2.72 \\
\hline MP-290 & $\mathrm{Kr}$ ? & $37^{\circ} 42^{\prime}$ & $119^{\circ} 15^{\prime}$ & 48.6 & 23.7 & 19.7 & 7.9 & 2.665 \\
\hline MP-293B & $\mathbf{K r}$ ? & $37^{\circ} 41^{\prime}$ & $119^{\circ} 16^{\prime}$ & 54.7 & 15.4 & 22.4 & 7.5 & 2.69 \\
\hline MP-298 & $\mathrm{Ktl}$ & $37^{\circ} 38^{\prime}$ & $119^{\circ} 21^{\prime}$ & 34.9 & 24.5 & 33.0 & 7.6 & 2.65 \\
\hline MP-304 & $\mathbf{K r}$ & $37^{\circ} 39^{\prime}$ & $119^{\circ} 22^{\prime}$ & 49.4 & 17.1 & 22.9 & 10.6 & 2.68 \\
\hline MP-305 & $\mathbf{K r}$ & $37^{\circ} 39^{\prime}$ & $119^{\circ} 21^{\prime}$ & 45.8 & 20.0 & 25.8 & 8.4 & 2.665 \\
\hline MP-306 & $\mathbf{K r}$ & $37^{\circ} 40^{\prime}$ & $119^{\circ} 21^{\prime}$ & 41.3 & 24.3 & 26.6 & 7.8 & 2.66 \\
\hline $\mathrm{MP}-307 \mathrm{~A}$ & $\mathrm{Ktl}$ & $37^{\circ} 41^{\prime}$ & $119^{\circ} 21^{\prime}$ & 38.5 & 24.1 & 31.7 & 5.7 & 2.67 \\
\hline MP-308 & Khd & $37^{\circ} 41^{\prime}$ & $119^{\circ} 20^{\prime}$ & 49.7 & 20.5 & 19.8 & 10.0 & 2.694 \\
\hline MP-309 & Kpp & $37^{\circ} 38^{\prime}$ & $119^{\circ} 22^{\prime}$ & 37.3 & 30.3 & 26.9 & 5.5 & 2.651 \\
\hline MP-311 & $\mathrm{Kpp}$ & $37^{\circ} 38^{\prime}$ & $119^{\circ} 22^{\prime}$ & 23.1 & 38.7 & 33.8 & 4.4 & 2.632 \\
\hline MP-322 & $\mathbf{K r}$ & $37^{\circ} 39^{\prime}$ & $119^{\circ} 20^{\prime}$ & 44.4 & 16.4 & 29.0 & 10.2 & 2.68 \\
\hline MP-328 & $\mathrm{Ktl}$ & $37^{\circ} 38^{\prime}$ & $119^{\circ} 21^{\prime}$ & 42.7 & 21.3 & 30.0 & 6.0 & 2.66 \\
\hline MP-332B & Khd & $37^{\circ} 40^{\prime}$ & $119^{\circ} 19^{\prime}$ & 47.9 & 22.2 & 19.8 & 10.1 & nd. \\
\hline MP-333 & $\mathbf{K j}$ & $37^{\circ} 40^{\prime}$ & $119^{\circ} 18^{\prime}$ & 39.2 & 27.2 & 25.1 & 8.6 & 2.67 \\
\hline MP-334 & Khd & $37^{\circ} 41^{\prime}$ & $119^{\circ} 19^{\prime}$ & 35.5 & 27.1 & 28.1 & 9.2 & 2.662 \\
\hline MP-335 & $\mathbf{K r}$ & $37^{\circ} 41^{\prime}$ & $119^{\circ} 18^{\prime}$ & 49.7 & 15.9 & 23.2 & 11.2 & - \\
\hline MP-337 & $\mathbf{K j}$ & $37^{\circ} 41^{\prime}$ & $119^{\circ} 18^{\prime}$ & 47.0 & 20.2 & 22.7 & 10.1 & 2.675 \\
\hline MP-338 & $\mathbf{K r}$ & $37^{\circ} 41^{\prime}$ & $119^{\circ} 18^{\prime}$ & 39.2 & 19.2 & 28.3 & 13.3 & 2.68 \\
\hline MP-340 _ & Ktl & $37^{\circ} 39^{\prime}$ & $119^{\circ} 21^{\prime}$ & 36.9 & 28.2 & 29.4 & 5.5 & 2.65 \\
\hline MP-341 & $\mathrm{Ktl}$ & $37^{\circ} 41^{\prime}$ & $119^{\circ} 21^{\prime}$ & 37.3 & 27.1 & 28.8 & 6.8 & 2.66 \\
\hline
\end{tabular}


TABLE 5.-Modes, specific gravity, and location of plutonic rocks-Continued

\begin{tabular}{|c|c|c|c|c|c|c|c|c|}
\hline Sample no. & $\begin{array}{l}\text { Map symbol } \\
\text { for rock } \\
\text { unit }\end{array}$ & $\begin{array}{c}\text { Latitude } \\
37^{\circ} 30^{\prime}-45^{\prime}\end{array}$ & $\begin{array}{l}\text { Longitude } \\
119^{\circ} 15^{\prime}-30^{\prime}\end{array}$ & $\begin{array}{c}\text { Plagioclase } \\
\text { (volume percent) }\end{array}$ & $\begin{array}{c}\text { Alkali } \\
\text { feldspar } \\
\text { (volume percent) }\end{array}$ & $\begin{array}{c}\text { Quartz } \\
\text { (volume percent) }\end{array}$ & $\begin{array}{c}\text { Mafic } \\
\text { minerals } \\
\text { (volume percent) }\end{array}$ & $\begin{array}{l}\text { Specific } \\
\text { gravity } \\
\left(\mathrm{g}^{\prime} / \mathrm{cm}^{3}\right)\end{array}$ \\
\hline MP-342 & Khd & $37^{\circ} 41^{\prime}$ & $119^{\circ} 21^{\prime}$ & 47.8 & 15.1 & 26.5 & 10.6 & 2.707 \\
\hline MP-348 & Kqd & $37^{\circ} 30^{\prime}$ & $119^{\circ} 26^{\prime}$ & 44.6 & 14.2 & 18.0 & 23.2 & 2.726 \\
\hline MP-351 & Ko? & $37^{\circ} 31^{\prime}$ & $119^{\circ} 27^{\prime}$ & 34.8 & 29.5 & 30.6 & 5.1 & 2.651 \\
\hline MP-353A & Ko & $37^{\circ} 31^{\prime}$ & $119^{\circ} 25^{\prime}$ & 39.2 & 18.9 & 33.4 & 8.5 & 2.683 \\
\hline MP-354A & $\mathrm{Ki}$ & $37^{\circ} 32^{\prime}$ & $119^{\circ} 25^{\prime}$ & 42.7 & 11.0 & 26.7 & 19.6 & 2.731 \\
\hline MP-361 & Ksp & $37^{\circ} 32^{\prime}$ & $119^{\circ} 28^{\prime}$ & 28.4 & 35.0 & 35.0 & 1.6 & 2.615 \\
\hline MP-364 & $\mathrm{Ki}$ & $37^{\circ} 32$ & $119^{\circ} 26^{\prime}$ & 42.9 & 10.9 & 25.1 & 21.1 & 2.73 \\
\hline MP-365 & Ko & $37^{\circ} 32^{\prime}$ & $119^{\circ} 26^{\prime}$ & 43.1 & 18.9 & 31.0 & 7.0 & 2.67 \\
\hline MP-366 & Ko & $37^{\circ} 32^{\prime}$ & $119^{\circ} 25^{\prime}$ & 43.8 & 17.5 & 33.4 & 5.3 & 2.655 \\
\hline MP-368 & Kpp? & $37^{\circ} 37^{\prime}$ & $119^{\circ} 23^{\prime}$ & 32.8 & 32.3 & 30.0 & 4.9 & 2.637 \\
\hline MP-371 & $\mathrm{Kj}$ & $37^{\circ} 37^{\prime}$ & $119^{\circ} 22^{\prime}$ & 28.8 & 33.0 & 36.2 & 2.0 & 2.627 \\
\hline MP-381 _ & $\mathrm{Kj}$ & $37^{\circ} 37^{\prime}$ & $119^{\circ} 23^{\prime}$ & 43.4 & 21.5 & 25.7 & 9.4 & 2.675 \\
\hline MP-385 & Klg & $37^{\circ} 36^{\prime}$ & $119^{\circ} 23^{\prime}$ & 26.5 & 47.9 & 23.1 & 2.5 & 2.625 \\
\hline MP-386 & $\overrightarrow{\mathrm{Kj}}$ & $37^{\circ} 36^{\prime}$ & $119^{\circ} 23^{\prime}$ & 49.4 & 16.4 & 24.5 & 9.7 & 2.675 \\
\hline MP-387 & Khe & $37^{\circ} 36^{\prime}$ & $119^{\circ} 23^{\prime}$ & 50.5 & 18.9 & 20.7 & 9.9 & 2.70 \\
\hline MP-388 & $\mathrm{Kj}$ & $37^{\circ} 36^{\prime}$ & $119^{\circ} 24^{\prime}$ & 51.4 & 17.3 & 22.6 & 8.7 & 2.675 \\
\hline MP-389B & $\mathrm{Kqd}$ & $37^{\circ} 37^{\prime}$ & $119^{\circ} 25^{\prime}$ & 59.2 & 0.1 & 8.1 & 32.6 & 2.835 \\
\hline MP-391 & Ko & $37^{\circ} 37^{\prime}$ & $119^{\circ} 26^{\prime}$ & 39.5 & 17.4 & 27.3 & 15.8 & 2.717 \\
\hline MP-392 & Ko & $37^{\circ} 37^{\prime}$ & $119^{\circ} 26^{\prime}$ & 40.8 & 21.0 & 26.3 & 11.9 & 2.68 \\
\hline MP-395 & Khe & $37^{\circ} 36^{\prime}$ & $119^{\circ} 23^{\prime}$ & 54.4 & 15.3 & 19.6 & 10.7 & 2.71 \\
\hline MP-398 & $\mathrm{Kj}$ & $37^{\circ} 35^{\prime}$ & $119^{\circ} 23^{\prime}$ & 45.6 & 15.9 & 26.2 & 12.3 & 2.675 \\
\hline MP-402 & Kgc & $37^{\circ} 31^{\prime}$ & $119^{\circ} 28^{\prime}$ & 53.6 & 6.1 & 25.8 & 14.5 & 2.73 \\
\hline $\mathrm{MP}-420 \mathrm{~A}$ & Kqd & $37^{\circ} 38^{\prime}$ & $119^{\circ} 22^{\prime}$ & 58.0 & 4.3 & 19.0 & 18.7 & 2.76 \\
\hline MP-429 & $\mathrm{Kj}$ & $37^{\circ} 37^{\prime}$ & $119^{\circ} 24^{\prime}$ & 49.0 & 19.1 & 27.4 & 4.5 & 2.69 \\
\hline $\mathrm{MP}-430 \mathrm{~A}$ & $\mathrm{Ki}$ & $37^{\circ} 37^{\prime}$ & $119^{\circ} 25^{\prime}$ & 49.1 & 3.8 & 19.5 & 27.6 & 2.78 \\
\hline MP-434A & $\mathrm{Krp}$ & $37^{\circ} 37^{\prime}$ & $119^{\circ} 24^{\prime}$ & 43.6 & 23.2 & 28.9 & 4.3 & 2.656 \\
\hline MP-437A & $\mathrm{Ko}$ & $37^{\circ} 38^{\prime}$ & $119^{\circ} 25^{\prime}$ & 52.0 & 10.4 & 21.5 & 16.1 & 2.723 \\
\hline MP-439A & $\mathrm{Kb}$ & $37^{\circ} 35^{\prime}$ & $119^{\circ} 24^{\prime}$ & 49.6 & 15.6 & 28.0 & 6.8 & 2.68 \\
\hline $\mathrm{MP}-440 \mathrm{~A}$ & $\mathbf{K b}$ & $37^{\circ} 35^{\prime}$ & $119^{\circ} 24^{\prime}$ & 44.7 & 21.3 & 31.2 & 2.8 & 2.657 \\
\hline MP-441 & $\mathrm{Ki}$ & $37^{\circ} 35^{\prime}$ & $119^{\circ} 25^{\prime}$ & 45.0 & 14.4 & 24.8 & 15.8 & 2.72 \\
\hline $\mathrm{MP}-443$ & Ko & $37^{\circ} 35^{\prime}$ & $119^{\circ} 27^{\prime}$ & 42.7 & 19.8 & 30.3 & 7.2 & 2.654 \\
\hline $\mathrm{MP}-447 \mathrm{~A}$ & Ko & $37^{\circ} 36^{\prime}$ & $119^{\circ} 29^{\prime}$ & 40.3 & 23.1 & 31.6 & 5.0 & 2.645 \\
\hline MP-448 & Ko & $37^{\circ} 36^{\prime}$ & $119^{\circ} 30^{\prime}$ & 40.5 & 21.9 & 33.8 & 3.8 & 2.659 \\
\hline MP-450 & Ko & $37^{\circ} 35^{\prime}$ & $119^{\circ} 29^{\prime}$ & 41.7 & 22.4 & 29.8 & 6.1 & 2.662 \\
\hline MP-453 & Ko & $37^{\circ} 35^{\prime}$ & $119^{\circ} 26^{\prime}$ & 42.1 & 21.9 & 28.0 & 8.0 & 2.636 \\
\hline MP-455 & $\mathrm{Ki}$ & $37^{\circ} 35^{\prime}$ & $119^{\circ} 26^{\prime}$ & 41.7 & 16.7 & 27.0 & 14.6 & 2.707 \\
\hline $\mathrm{MP}-460 \mathrm{~A}$ & $\mathrm{Kj}$ & $37^{\circ} 34^{\prime}$ & $119^{\circ} 23^{\prime}$ & 50.5 & 17.9 & 21.3 & 10.3 & 2.68 \\
\hline MP-465 & $\mathrm{Kb}$ & $37^{\circ} 34^{\prime}$ & $119^{\circ} 24^{\prime}$ & 45.7 & 18.4 & 31.3 & 4.6 & 2.667 \\
\hline MP-470A & Ko & $37^{\circ} 34^{\prime}$ & $119^{\circ} 25^{\prime}$ & 41.9 & 23.0 & 27.7 & 7.4 & 2.655 \\
\hline MP-472 & $\mathbf{K i}$ & $37^{\circ} 33^{\prime}$ & $119^{\circ} 24^{\prime}$ & 35.0 & 27.0 & 27.2 & 10.8 & 2.673 \\
\hline MP-473 & Ko & $37^{\circ} 33^{\prime}$ & $119^{\circ} 25^{\prime}$ & 39.2 & 23.9 & 31.6 & 5.2 & 2.64 \\
\hline MP-475 & Ko & $37^{\circ} 33^{\prime}$ & $119^{\circ} 27^{\prime}$ & 37.0 & 27.1 & 30.3 & 5.6 & 2.64 \\
\hline $\mathrm{MP}-478$ & $\mathrm{Ki}$ & $37^{\circ} 33^{\prime}$ & $119^{\circ} 29^{\prime}$ & 42.6 & 12.4 & 26.5 & 18.5 & 2.74 \\
\hline MP-484 & $\mathrm{Kj}$ & $37^{\circ} 33^{\prime}$ & $119^{\circ} 24^{\prime}$ & 54.3 & 13.9 & 16.4 & 15.4 & 2.70 \\
\hline MP-486 & Krp & $37^{\circ} 32^{\prime}$ & $119^{\circ} 24^{\prime}$ & 35.8 & 27.2 & 33.2 & 3.8 & 2.637 \\
\hline MP-497 _ _ & Krp & $37^{\circ} 40^{\prime}$ & $119^{\circ} 25^{\prime}$ & 34.7 & 31.7 & 30.2 & 3.4 & 2.64 \\
\hline MP-498 & $\mathrm{Kgr}$ & $37^{\circ} 40^{\prime}$ & $119^{\circ} 26^{\prime}$ & 50.6 & 7.9 & 18.9 & 22.6 & 2.77 \\
\hline MP-499A & Kgr & $37^{\circ} 40^{\prime}$ & $119^{\circ} 26^{\prime}$ & 58.2 & 4.6 & 14.8 & 22.4 & 2.787 \\
\hline MP-502 & Ko & $37^{\circ} 35^{\prime}$ & $119^{\circ} 28^{\prime}$ & 40.2 & 20.2 & 27.5 & 12.1 & 2.683 \\
\hline MP-505 & Ko & $37^{\circ} 36^{\prime}$ & $119^{\circ} 27^{\prime}$ & 38.0 & 23.6 & 30.0 & 8.4 & 2.677 \\
\hline MP-506 & Ko & $37^{\circ} 37^{\prime}$ & $119^{\circ} 27^{\prime}$ & 36.4 & 25.9 & 30.5 & 7.2 & 2.64 \\
\hline MP-510 & Kqd & $37^{\circ} 36^{\prime}$ & $119^{\circ} 29^{\prime}$ & 50.3 & 2.4 & 24.0 & 23.4 & 2.172 \\
\hline MP-511B & Ko & $37^{\circ} 35^{\prime}$ & $119^{\circ} 29^{\prime}$ & 39.3 & 23.9 & 31.0 & 5.8 & 2.64 \\
\hline MP-512 & Ko & $37^{\circ} 35^{\prime}$ & $119^{\circ} 29^{\prime}$ & 42.9 & 21.1 & 30.2 & 5.8 & 2.67 \\
\hline MP-514 & $\mathrm{Kqd}$ & $37^{\circ} 34^{\prime}$ & $119^{\circ} 28^{\prime}$ & 55.3 & 0.2 & 10.8 & 33.7 & 2.812 \\
\hline MP-520 & $\mathbf{K j}$ & $37^{\circ} 35^{\prime}$ & $119^{\circ} 24^{\prime}$ & 47.3 & 19.2 & 23.5 & 10.0 & 2.68 \\
\hline MP-521 & $\mathrm{Ki}$ & $37^{\circ} 34^{\prime}$ & $119^{\circ} 24^{\prime}$ & 45.9 & 12.2 & 25.6 & 16.3 & 2.717 \\
\hline MP-525 & $\mathrm{Ki}$ & $37^{\circ} 34^{\prime}$ & $119^{\circ} 24^{\prime}$ & 40.6 & 8.6 & 30.8 & 20.0 & 2.727 \\
\hline MP-529 & $\mathrm{Ki}$ & $37^{\circ} 34^{\prime}$ & $119^{\circ} 25^{\prime}$ & 44.8 & 10.2 & 28.2 & 16.8 & 2.714 \\
\hline MP-531 & Ko & $37^{\circ} 34^{\prime}$ & $119^{\circ} 26^{\prime}$ & 40.8 & 21.0 & 30.6 & 7.5 & 2.638 \\
\hline MP-532 & Ko & $37^{\circ} 34^{\prime}$ & $119^{\circ} 27^{\prime}$ & 40.1 & 23.6 & 30.0 & 6.3 & 2.631 \\
\hline MP-533 & Ko & $37^{\circ} 33^{\prime}$ & $119^{\circ} 27^{\prime}$ & 39.0 & 24.5 & 30.4 & 6.1 & 2.658 \\
\hline MP-534 & Ko & $37^{\circ} 34^{\prime}$ & $119^{\circ} 27^{\prime}$ & 41.6 & 25.0 & 28.3 & 5.1 & 2.658 \\
\hline MP-536 & $\mathrm{Ki}$ & $37^{\circ} 34^{\prime}$ & $119^{\circ} 25^{\prime}$ & 45.9 & 9.3 & 29.3 & 15.4 & 2.71 \\
\hline MP-539 & Ko & $37^{\circ} 34^{\prime}$ & $119^{\circ} 28^{\prime}$ & 37.3 & 24.4 & 32.0 & 6.3 & 2.66 \\
\hline MP-540 & $\mathrm{Ki}$ & $37^{\circ} 33^{\prime}$ & $119^{\circ} 29^{\prime}$ & 49.8 & 8.5 & 23.1 & 18.6 & 2.75 \\
\hline MP-541 & $\mathrm{Kb}$ & $37^{\circ} 34^{\prime}$ & $119^{\circ} 24^{\prime}$ & 48.4 & 17.2 & 28.7 & 5.7 & 2.675 \\
\hline
\end{tabular}


TABLE 5.-Modes, specific gravity, and location of plutonic rocks-Continued

\begin{tabular}{|c|c|c|c|c|c|c|c|c|}
\hline Sample no. & $\begin{array}{c}\text { Map symbol } \\
\text { for rock } \\
\text { unit }\end{array}$ & $\begin{array}{c}\text { Latitude } \\
37^{\circ} 3 \theta^{\prime}-45^{\prime}\end{array}$ & $\begin{array}{c}\text { Longitude } \\
119^{\circ} 15^{\prime}-30^{\prime}\end{array}$ & $\begin{array}{c}\text { Plagioclase } \\
\text { (volume percent) }\end{array}$ & $\begin{array}{c}\text { Alkali } \\
\text { feldspar } \\
\text { (volume percent) }\end{array}$ & $\begin{array}{c}\text { Quartz } \\
\text { (volume percent) }\end{array}$ & $\begin{array}{c}\text { Mafic } \\
\text { minerals } \\
\text { (volume percent) }\end{array}$ & $\begin{array}{l}\text { Specific } \\
\text { gravity } \\
\left(\mathrm{g} / \mathrm{cm}^{3}\right)\end{array}$ \\
\hline MP-543 _- & $\mathbf{K i}$ & $37^{\circ} 32^{\prime}$ & $119^{\circ} 25^{\prime}$ & 39.8 & 15.9 & 25.9 & 18.3 & 2.715 \\
\hline MP-546 & $\overrightarrow{\mathbf{K i}}$ & $37^{\circ} 31^{\prime}$ & $119^{\circ} 24^{\prime}$ & 36.1 & 23.5 & 22.1 & 18.2 & 2.715 \\
\hline MP-547 _ & Ko & $37^{\circ} 32^{\prime}$ & $119^{\circ} 26^{\prime}$ & 39.5 & 21.7 & 32.0 & 6.8 & 2.64 \\
\hline MP-549 & $\mathrm{Krp}$ & $37^{\circ} 39^{\prime}$ & $119^{\circ} 25^{\prime}$ & 34.8 & 29.1 & 30.7 & 5.4 & 2.651 \\
\hline MP-557A - & $\mathrm{Krp}$ & $37^{\circ} 39^{\prime}$ & $119^{\circ} 24^{\prime}$ & 28.7 & 32.3 & 36.3 & 2.7 & 2.636 \\
\hline MP-559 & $\mathbf{K j}$ & $37^{\circ} 40^{\prime}$ & $119^{\circ} 24^{\prime}$ & 44.6 & 19.8 & 25.3 & 10.3 & 2.70 \\
\hline MP-560 _ & $\mathbf{K i}$ & $37^{\circ} 38^{\prime}$ & $119^{\circ} 26^{\prime}$ & 44.4 & 6.8 & 25.4 & 23.4 & 2.75 \\
\hline MP-562A & $\mathbf{K i}$ & $37^{\circ} 39^{\prime}$ & $119^{\circ} 28^{\prime}$ & 46.6 & 4.9 & 26.5 & 21.9 & 2.74 \\
\hline MP-563 _ _ & $\mathbf{K i}$ & $37^{\circ} 38^{\prime}$ & $119^{\circ} 26^{\prime}$ & 43.7 & 9.9 & 26.6 & 19.8 & 2.74 \\
\hline MP-565 & $\overrightarrow{\mathrm{Ki}}$ & $37^{\circ} 38^{\prime}$ & $119^{\circ} 28^{\prime}$ & 43.9 & 12.6 & 25.8 & 17.6 & 2.715 \\
\hline MP-567 _-_ & Ko & $37^{\circ} 38^{\prime}$ & $119^{\circ} 27^{\prime}$ & 40.6 & 22.7 & 27.2 & 9.5 & 2.65 \\
\hline MP-568 & $\mathbf{K i}$ & $37^{\circ} 38^{\prime}$ & $119^{\circ} 25^{\prime}$ & 48.4 & 11.2 & 23.4 & 17.0 & 2.735 \\
\hline MP-569A & $\mathrm{Kmc}$ & $37^{\circ} 41^{\prime}$ & $119^{\circ} 25^{\prime}$ & 29.0 & 34.0 & 35.2 & 1.8 & 2.63 \\
\hline MP-572 & Ko & $37^{\circ} 36^{\prime}$ & $119^{\circ} 28^{\prime}$ & 39.6 & 26.3 & 24.6 & 8.5 & 2.66 \\
\hline MP-573 _- & Ko & $37^{\circ} 36^{\prime}$ & $119^{\circ} 28^{\prime}$ & 37.0 & 27.0 & 28.8 & 7.2 & 2.653 \\
\hline MP-574 & Ko & $37^{\circ} 36^{\prime}$ & $119^{\circ} 29^{\prime}$ & 38.9 & 25.4 & 30.5 & 5.2 & 2.653 \\
\hline MP-575 _- & Ko & $37^{\circ} 36^{\prime}$ & $119^{\circ} 29^{\prime}$ & 37.5 & 25.1 & 31.6 & 5.8 & 2.66 \\
\hline MP-576 & Ko & $37^{\circ} 37^{\prime}$ & $119^{\circ} 28^{\prime}$ & 36.0 & 24.5 & 30.9 & 8.6 & 2.65 \\
\hline MP-577 _ & $\mathrm{Krp}$ & $37^{\circ} 38^{\prime}$ & $119^{\circ} 25^{\prime}$ & 32.9 & 35.6 & 28.6 & 2.9 & 2.645 \\
\hline MP-578 & $\mathrm{Kj}^{2}$ & $37^{\circ} 38^{\prime}$ & $119^{\circ} 25^{\prime}$ & 52.9 & 12.2 & 19.7 & 15.2 & 2.717 \\
\hline MP-585 & $\mathbf{K i}$ & $37^{\circ} 39^{\prime}$ & $119^{\circ} 29^{\prime}$ & 49.9 & 3.9 & 27.0 & 19.2 & 2.75 \\
\hline MP-586A & Ko & $37^{\circ} 38^{\prime}$ & $119^{\circ} 29^{\prime}$ & 44.8 & 11.7 & 29.7 & 13.7 & 2.71 \\
\hline MP-591 & $\mathbf{K i}$ & $37^{\circ} 34^{\prime}$ & $119^{\circ} 29^{\prime}$ & 49.1 & 8.2 & 26.3 & 16.4 & 2.73 \\
\hline MP-602A & $\mathrm{Kgp}$ & $37^{\circ} 40^{\prime}$ & $119^{\circ} 25^{\prime}$ & 35.4 & 30.0 & 29.9 & 4.7 & 2.647 \\
\hline MP-603B & $\mathrm{Kgp}$ & $37^{\circ} 41^{\prime}$ & $119^{\circ} 26^{\prime}$ & 45.0 & 14.6 & 26.5 & 13.9 & 2.70 \\
\hline MP-605A _- & $\mathrm{Kgr}$ & $37^{\circ} 40^{\prime}$ & $119^{\circ} 26^{\prime}$ & 53.9 & 6.9 & 13.4 & 25.8 & 2.80 \\
\hline MP-608A & Krp & $37^{\circ} 38^{\prime}$ & $119^{\circ} 25^{\prime}$ & 34.3 & 32.7 & 28.6 & 4.4 & 2.65 \\
\hline MP-615A & Krp & $37^{\circ} 39^{\prime}$ & $119^{\circ} 25^{\prime}$ & 30.9 & 35.1 & 29.8 & 4.2 & 2.625 \\
\hline MP-617 _ & Ko ${ }^{-}$ & $37^{\circ} 37^{\prime}$ & $119^{\circ} 27^{\prime}$ & 39.2 & 23.6 & 29.1 & 8.1 & nd \\
\hline MP-619 _-_ & Ko? & $37^{\circ} 31^{\prime}$ & $119^{\circ} 26^{\prime}$ & 30.0 & 30.0 & 34.6 & 5.4 & 2.635 \\
\hline MP-622 & Ksp & $37^{\circ} 33^{\prime}$ & $119^{\circ} 30^{\prime}$ & 30.0 & 30.1 & 31.4 & 8.6 & 2.66 \\
\hline MP-623 & $\mathrm{Ke}$ & $37^{\circ} 33^{\prime}$ & $119^{\circ} 30^{\prime}$ & 37.3 & 24.6 & 30.3 & 7.8 & 2.658 \\
\hline MP-629 & Ksp & $37^{\circ} 32^{\prime}$ & $119^{\circ} 28^{\prime}$ & 30.8 & 35.5 & 32.5 & 1.2 & 2.616 \\
\hline MP-632 & $\mathrm{Kj}^{2}$ & $37^{\circ} 31^{\prime}$ & $119^{\circ} 23^{\prime}$ & 42.2 & 26.6 & 23.7 & 7.5 & 2.67 \\
\hline MP-640 & Ktk & $37^{\circ} 35^{\prime}$ & $119^{\circ} 19^{\prime}$ & 35.0 & 33.4 & 29.3 & 2.3 & 2.613 \\
\hline MP-643A _- & $\mathrm{Kn}$ & $37^{\circ} 31^{\prime}$ & $119^{\circ} 20^{\prime}$ & 30.5 & 36.7 & 32.5 & 0.3 & 2.62 \\
\hline MP-644 & $\mathrm{Kn}$ & $37^{\circ} 32^{\prime}$ & $119^{\circ} 19^{\prime}$ & 24.7 & 43.9 & 29.1 & 2.2 & 2.604 \\
\hline MP-675 _ & Khd & $37^{\circ} 41^{\prime}$ & $119^{\circ} 28^{\prime}$ & 43.8 & 14.2 & 25.4 & 16.6 & 2.71 \\
\hline MP-676 & Khd & $37^{\circ} 42^{\prime}$ & $119^{\circ} 28^{\prime}$ & 36.0 & 26.2 & 30.9 & 6.8 & 2.66 \\
\hline MP-677 & Khd & $37^{\circ} 42^{\prime}$ & $119^{\circ} 29^{\prime}$ & 43.9 & 25.7 & 20.8 & 9.6 & 2.675 \\
\hline MP-678 & Khd & $37^{\circ} 44^{\prime}$ & $119^{\circ} 23^{\prime}$ & 47.7 & 20.1 & 24.7 & 7.5 & 2.66 \\
\hline MP-680 & Khd & $37^{\circ} 43^{\prime}$ & $119^{\circ} 22^{\prime}$ & 49.1 & 19.9 & 24.7 & 6.3 & 2.68 \\
\hline MP-682 & $\mathrm{Ktl}$ & $37^{\circ} 42^{\prime}$ & $119^{\circ} 20^{\prime}$ & 35.2 & 28.9 & 31.3 & .4 .6 & 2.64 \\
\hline MP-684 & Khd & $37^{\circ} 45^{\prime}$ & $119^{\circ} 23^{\prime}$ & 46.4 & 20.4 & 26.5 & 6.7 & 2.67 \\
\hline MP-685 & Khd & $37^{\circ} 45^{\prime}$ & $119^{\circ} 22^{\prime}$ & 45.0 & 20.3 & 25.7 & 9.0 & 2.67 \\
\hline MP-688 & Khd & $37^{\circ} 45^{\prime}$ & $119^{\circ} 23^{\prime}$ & 47.4 & 19.6 & 26.2 & 6.8 & 2.675 \\
\hline MP-690 & Khd & $37^{\circ} 45^{\prime}$ & $119^{\circ} 29^{\prime}$ & 44.4 & 25.8 & 21.5 & 8.2 & 2.67 \\
\hline MP-691 & Kec & $37^{\circ} 45^{\prime}$ & $119^{\circ} 22^{\prime}$ & 37.5 & 28.1 & 29.7 & 4.7 & 2.65 \\
\hline MP-692 & Kec & $37^{\circ} 45^{\prime}$ & $119^{\circ} 22^{\prime}$ & 41.7 & 26.1 & 26.4 & 5.8 & $2.66+$ \\
\hline MP-693 & $\mathrm{Ktl}$ & $37^{\circ} 45^{\prime}$ & $119^{\circ} 21^{\prime}$ & 40.0 & 19.3 & 35.5 & 5.2 & 2.65 \\
\hline MP-694 & $\mathrm{Kr}$ & $37^{\circ} 45^{\prime}$ & $119^{\circ} 20^{\prime}$ & 35.8 & 28.1 & 29.8 & 6.3 & $2.65-$ \\
\hline MP-695 & $\mathrm{Kr}$ & $37^{\circ} 45^{\prime}$ & $119^{\circ} 20^{\prime}$ & 32.9 & 26.2 & 34.7 & 6.2 & 2.64 \\
\hline MP-698 & Khd & $37^{\circ} 43^{\prime}$ & $119^{\circ} 24^{\prime}$ & 37.4 & 27.5 & 25.1 & 10.0 & 2.675 \\
\hline MP-702 & Khd & $37^{\circ} 44^{\prime}$ & $119^{\circ} 25^{\prime}$ & 48.7 & 20.0 & 22.1 & 9.2 & 2.68 \\
\hline MP-703 & Khd & $37^{\circ} 44^{\prime}$ & $119^{\circ} 27^{\prime}$ & 49.2 & 13.2 & 25.3 & 12.4 & 2.69 \\
\hline MP-708 & Khd & $37^{\circ} 42^{\prime}$ & $119^{\circ} 24^{\prime}$ & 49.4 & 19.0 & 21.9 & 9.7 & $2.70+$ \\
\hline MP-712 & Kme & $37^{\circ} 42^{\prime}$ & $119^{\circ} 25^{\prime}$ & 28.7 & 32.9 & 36.6 & 1.8 & 2.61 \\
\hline MP-713 & $\mathrm{Kr}$ & $37^{\circ} 42^{\prime}$ & $119^{\circ} 24^{\prime}$ & 49.1 & 16.4 & 23.3 & 11.2 & $2.67-$ \\
\hline MP-714 & $\mathrm{Kr}$ & $37^{\circ} 40^{\prime}$ & $119^{\circ} 23^{\prime}$ & 47.8 & 11.0 & 29.0 & 12.2 & $2.69+$ \\
\hline MP-715 _ _ & $\mathbf{K r}$ & $37^{\circ} 41^{\prime}$ & $119^{\circ} 22^{\prime}$ & 40.0 & 23.6 & 28.9 & 7.5 & 2.66 \\
\hline MP-716 & $\mathbf{K r}$ & $37^{\circ} 41^{\prime}$ & $119^{\circ} 23^{\prime}$ & 39.1 & 24.5 & 28.8 & 7.6 & $2.65-2.66$ \\
\hline MP-717 & $\mathbf{K r}$ & $37^{\circ} 41^{\prime}$ & $119^{\circ} 23^{\prime}$ & 48.8 & 16.8 & 24.6 & 9.8 & 2.67 \\
\hline MP-718 & $\mathbf{K r}$ & $37^{\circ} 44^{\prime}$ & $119^{\circ} 19^{\prime}$ & 33.4 & 30.2 & 31.2 & 5.2 & 2.64 \\
\hline MP-723 & $\mathbf{K r}$ & $37^{\circ} 43^{\prime}$ & $119^{\circ} 16^{\prime}$ & 50.6 & 15.7 & 21.8 & 11.9 & $2.68+$ \\
\hline MP-724 & $\mathbf{K r}$ & $37^{\circ} 43^{\prime}$ & $119^{\circ} 16^{\prime}$ & 49.3 & 14.6 & 26.2 & 9.9 & $2.69-$ \\
\hline MP-728 & $\mathbf{K r}$ & $37^{\circ} 43^{\prime}$ & $119^{\circ} 17^{\prime}$ & 48.4 & 16.7 & 23.0 & 11.9 & 2.69 \\
\hline MP-729 _-_- & $\mathbf{K r}$ & $37^{\circ} 43^{\prime}$ & $119^{\circ} 17^{\prime}$ & 51.4 & 10.9 & 26.4 & 11.3 & 2.69 \\
\hline MP-730 & $\mathbf{K r}$ & $37^{\circ} 43^{\prime}$ & $119^{\circ} 18^{\prime}$ & 39.3 & 25.1 & 29.6 & 6.0 & 2.66 \\
\hline
\end{tabular}


TABLE 5.-Modes, specific gravity, and location of plutonic rocks-Continued

\begin{tabular}{|c|c|c|c|c|c|c|c|c|}
\hline Sample no. & $\begin{array}{l}\text { Map symbol } \\
\text { for rock } \\
\text { unit }\end{array}$ & $\begin{array}{l}\text { Latitude } \\
37^{\circ} 30^{\prime}-45^{\prime}\end{array}$ & $\begin{array}{l}\text { Longitude } \\
119^{\circ} 15^{\prime}-30^{\prime}\end{array}$ & $\begin{array}{c}\text { Plagioclase } \\
\text { (volume percent) }\end{array}$ & $\begin{array}{c}\text { Alkali } \\
\text { feldspar } \\
\text { (volume percent) }\end{array}$ & $\begin{array}{c}\text { Quartz } \\
\text { (volume percent) }\end{array}$ & $\begin{array}{c}\text { Mafic } \\
\text { minerals } \\
\text { (volume percent) }\end{array}$ & $\begin{array}{l}\text { Specific } \\
\text { gravity } \\
\left(\mathrm{g} / \mathrm{cm}^{3}\right)\end{array}$ \\
\hline $\mathrm{MP}-732$ & $\mathrm{Kr}$ & $37^{\circ} 44^{\prime}$ & $119^{\circ} 18^{\prime}$ & 42.5 & 23.2 & 27.0 & 7.3 & $2.67-$ \\
\hline MP-735 & $\mathrm{Kr}$ & $37^{\circ} 45^{\prime}$ & $119^{\circ} 18^{\prime}$ & 41.0 & 25.3 & 26.3 & 7.4 & 2.66 \\
\hline MP-737 & $\mathrm{Kr}$ & $37^{\circ} 44^{\prime}$ & $119^{\circ} 19^{\prime}$ & 35.6 & 27.5 & 30.5 & 6.4 & 2.65 \\
\hline MP-738 & $\mathrm{Kr}$ & $37^{\circ} 43^{\prime}$ & $119^{\circ} 19^{\prime}$ & 36.1 & 29.1 & 29.1 & 5.7 & $2.65-$ \\
\hline MP-739 & Ktl & $37^{\circ} 44^{\prime}$ & $119^{\circ} 20^{\prime}$ & 38.7 & 25.7 & 29.7 & 5.9 & 2.634 \\
\hline MP-741 & $\mathbf{K k}$ & $37^{\circ} 45^{\prime}$ & $119^{\circ} 15^{\prime}$ & 51.4 & 15.7 & 13.1 & 19.8 & 2.73 \\
\hline MP-753 & $\mathrm{Kmc}$ & $37^{\circ} 42^{\prime}$ & $119^{\circ} 26^{\prime}$ & 22.9 & 33.1 & 40.6 & 3.4 & 2.62 \\
\hline MP-755 & $\mathrm{Kmc}$ & $37^{\circ} 42^{\prime}$ & $119^{\circ} 26^{\prime}$ & 23.3 & 35.1 & 39.1 & 2.5 & 2.62 \\
\hline MP-759 _ & Khd & $37^{\circ} 43^{\prime}$ & $119^{\circ} 27^{\prime}$ & 49.6 & 17.0 & 22.6 & 10.8 & 2.69 \\
\hline MP-760 & Khd & $37^{\circ} 43^{\prime}$ & $119^{\circ} 26^{\prime}$ & 46.5 & 16.3 & 22.0 & 15.2 & 2.72 \\
\hline MP-761 & $\mathrm{Kgp}$ & $37^{\circ} 43^{\prime}$ & $119^{\circ} 25^{\prime}$ & 37.9 & 27.4 & 28.8 & 5.9 & 2.64 \\
\hline MP-762 & $\mathrm{Kr}$ & $37^{\circ} 40^{\prime}$ & $119^{\circ} 23^{\prime}$ & 45.7 & 15.8 & 25.0 & 13.5 & $2.67+$ \\
\hline MP-763 & $\mathrm{Kr}$ & $37^{\circ} 40^{\prime}$ & $119^{\circ} 23^{\prime}$ & 47.5 & 17.1 & 24.8 & 10.6 & $2.68-2.67$ \\
\hline MP-764 & $\mathrm{Kr}$ & $37^{\circ} 41^{\prime}$ & $119^{\circ} 23^{\prime}$ & 43.8 & 19.2 & 27.6 & 9.4 & 2.66 \\
\hline MP-765 _ _. & $\mathrm{Kr}$ & $37^{\circ} 41^{\prime}$ & $119^{\circ} 24^{\prime}$ & 47.2 & 19.5 & 23.0 & 10.3 & 2.69 \\
\hline MP-766 & $\mathrm{Kmc}$ & $37^{\circ} 41^{\prime}$ & $119^{\circ} 25^{\prime}$ & 29.8 & 31.0 & 37.2 & 2.0 & nd. \\
\hline MP-770 & $\mathrm{Kgp}$ & $37^{\circ} 42^{\prime}$ & $119^{\circ} 25^{\prime}$ & 38.9 & 23.2 & 30.2 & 7.8 & 2.66 \\
\hline MP-772 & $\mathrm{Kgp}$ & $37^{\circ} 41^{\prime}$ & $119^{\circ} 24^{\prime}$ & 28.2 & 25.8 & 38.3 & 7.7 & 2.65 \\
\hline MP-773 & $\mathrm{Kgp}$ & $37^{\circ} 41^{\prime}$ & $119^{\circ} 25^{\prime}$ & 38.4 & 25.0 & 27.9 & 8.7 & 2.66 \\
\hline MP-775 & $\mathrm{Kr}$ & $37^{\circ} 41^{\prime}$ & $119^{\circ} 24^{\prime}$ & 42.8 & 19.0 & 28.4 & 9.8 & 2.69 \\
\hline MP-776 & $\mathrm{Kgp}$ & $37^{\circ} 40^{\prime}$ & $119^{\circ} 24^{\prime}$ & 37.2 & 22.5 & 32.0 & 8.3 & 2.65 \\
\hline MP-779 & $\mathrm{Kgp}$ & $37^{\circ} 41^{\prime}$ & $119^{\circ} 25^{\prime}$ & 33.9 & 26.2 & 33.3 & 6.6 & 2.66 \\
\hline MP-786 & Ker & $37^{\circ} 40^{\prime}$ & $119^{\circ} 24^{\prime}$ & 47.0 & 0 & 5.3 & 47.7 & - \\
\hline MP-788 & $\mathbf{K r}$ & $37^{\circ} 40^{\prime}$ & $119^{\circ} 23^{\prime}$ & 49.1 & 17.3 & 25.0 & 8.6 & 2.67 \\
\hline MP-789 & $\mathrm{Kr}$ & $37^{\circ} 40^{\prime}$ & $119^{\circ} 23^{\prime}$ & 51.5 & 13.1 & 21.0 & 14.4 & 2.69 \\
\hline MP-790A & $\mathrm{Ktl}$ & $37^{\circ} 42^{\prime}$ & $119^{\circ} 23^{\prime}$ & 37.6 & 21.4 & 33.0 & 8.0 & 2.67 \\
\hline MP-792 & Khd & $37^{\circ} 45^{\prime}$ & $119^{\circ} 28^{\prime}$ & 46.2 & 25.6 & 20.6 & 7.6 & 2.69 \\
\hline MP-793 & Khd & $37^{\circ} 44^{\prime}$ & $119^{\circ} 29^{\prime}$ & 46.9 & 19.2 & 22.8 & 11.1 & 2.693 \\
\hline MP-794 & $\mathrm{Kr}$ & $37^{\circ} 43^{\prime}$ & $119^{\circ} 19^{\prime}$ & 34.4 & 28.0 & 30.0 & 7.6 & 2.65 \\
\hline MP-795B & Ktl & $37^{\circ} 43^{\prime}$ & $119^{\circ} 19^{\prime}$ & 43.9 & 22.7 & 27.9 & 5.5 & 2.644 \\
\hline MP-796 & Ktl & $37^{\circ} 42^{\prime}$ & $119^{\circ} 19^{\prime}$ & 38.2 & 22.8 & 32.1 & 6.9 & 2.64 \\
\hline MP-797 & $\mathrm{Kr}$ & $37^{\circ} 42^{\prime}$ & $119^{\circ} 19^{\prime}$ & 41.1 & 22.1 & 29.8 & 7.0 & $2.65-$ \\
\hline MP-798 & $\mathrm{Kr}$ & $37^{\circ} 42^{\prime}$ & $119^{\circ} 18^{\prime}$ & 43.6 & 19.2 & 26.5 & 10.7 & $2.68-$ \\
\hline MP-799 & $\mathrm{Kr}$ & $37^{\circ} 42^{\prime}$ & $119^{\circ} 18^{\prime}$ & 47.2 & 15.4 & 27.2 & 10.2 & 2.67 \\
\hline MP-801 & $\mathbf{K} \mathbf{j}$ & $37^{\circ} 42^{\prime}$ & $119^{\circ} 17^{\prime}$ & 66.6 & 7.4 & 12.4 & 13.6 & 2.70 \\
\hline MP-806 & $\mathrm{Kr}$ & $37^{\circ} 43^{\prime}$ & $119^{\circ} 16^{\prime}$ & 52.7 & 17.4 & 18.2 & 11.7 & $2.68-2.69$ \\
\hline MP-808 & $\mathrm{Kr}$ & $37^{\circ} 43^{\prime}$ & $119^{\circ} 17^{\prime}$ & 50.2 & 15.4 & 22.1 & 12.3 & 2.68 \\
\hline MP-811 _ & Kqd & $37^{\circ} 44^{\prime}$ & $119^{\circ} 16^{\prime}$ & 57.2 & 0.1 & 5.4 & 37.3 & 2.86 \\
\hline MP-814 & $\mathbf{K r}$ & $37^{\circ} \mathbf{4 4 ^ { \prime }}$ & $119^{\circ} 18^{\prime}$ & 40.4 & 20.6 & 31.1 & 7.9 & $2.65+$ \\
\hline MP-815 & $\mathrm{Kr}$ & $37^{\circ} 44^{\prime}$ & $119^{\circ} 17^{\prime}$ & 43.0 & 22.4 & 25.5 & 9.1 & 2.67 \\
\hline MP-816 & $\mathrm{Kk}$ & $37^{\circ} 45^{\prime}$ & $119^{\circ} 17^{\prime}$ & 63.4 & 9.6 & 12.0 & 15.0 & 2.77 \\
\hline MP-817 & $\mathrm{Kr}$ & $37^{\circ} 44^{\prime}$ & $119^{\circ} 17^{\prime}$ & 41.2 & 20.9 & 26.8 & 11.1 & $2.70-$ \\
\hline MP-818 & Ktl & $37^{\circ} 42^{\prime}$ & $119^{\circ} 20^{\prime}$ & 41.8 & 25.2 & 27.5 & 5.5 & 2.645 \\
\hline MP-819 & Khd & $37^{\circ} 44^{\prime}$ & $119^{\circ} 22^{\prime}$ & 43.7 & 24.0 & 25.2 & 7.1 & $2.66+$ \\
\hline MP-820 & Ktl & $37^{\circ} 43^{\prime}$ & $119^{\circ} 19^{\prime}$ & 37.2 & 23.9 & 33.1 & 5.8 & 2.65 \\
\hline MP-821 & Kec & $37^{\circ} 44^{\prime}$ & $119^{\circ} 21^{\prime}$ & 33.0 & 29.9 & 32.0 & 5.1 & $2.65-$ \\
\hline MP-822 & $\mathrm{Ktl}$ & $37^{\circ} \mathbf{4} 4^{\prime}$ & $119^{\circ} 21^{\prime}$ & 41.6 & 18.0 & 33.5 & 6.9 & 2.66 \\
\hline MP-823 & Ko & $37^{\circ} 36^{\prime}$ & $119^{\circ} 30^{\prime}$ & 39.2 & 24.1 & 28.9 & 7.8 & 2.65 \\
\hline MP-825 & $\mathbf{K} \mathbf{k}$ & $37^{\circ} 45^{\prime}$ & $119^{\circ} 15^{\prime}$ & 52.6 & 10.7 & 11.3 & 25.4 & 2.76 \\
\hline MP-825-2 & Ko & $37^{\circ} 35^{\prime}$ & $119^{\circ} 30^{\prime}$ & 39.5 & 27.2 & 25.8 & 7.5 & 2.648 \\
\hline MP-831 & $\mathrm{Kk}$ & $37^{\circ} 44^{\prime}$ & $119^{\circ} 15^{\prime}$ & 62.8 & 9.3 & 11.5 & 16.4 & 2.73 \\
\hline MP-833 & $\mathbf{K k}$ & $37^{\circ} 45^{\prime}$ & $119^{\circ} 15^{\prime}$ & 46.6 & 14.7 & 13.4 & 25.3 & 2.765 \\
\hline MP-835 & Kk & $37^{\circ} 44^{\prime}$ & $119^{\circ} 16^{\prime}$ & 65.0 & 1.3 & 5.9 & 27.8 & 2.79 \\
\hline MP-836 & $\mathrm{Kr}$ & $37^{\circ} 44^{\prime}$ & $119^{\circ} 16^{\prime}$ & 45.1 & 16.2 & 26.3 & 12.4 & $2.68+$ \\
\hline MP-843 & $\mathrm{Krp}$ & $37^{\circ} 39^{\prime}$ & $119^{\circ} 25^{\prime}$ & 30.7 & 35.6 & 31.3 & 2.4 & 2.625 \\
\hline MP-844 & $\mathrm{Krp}$ & $37^{\circ} 39^{\prime}$ & $119^{\circ} 25^{\prime}$ & 29.6 & 38.3 & 30.5 & 1.6 & 2.63 \\
\hline MP-845 & Krp & $37^{\circ} 39^{\prime}$ & $119^{\circ} 25^{\prime}$ & 37.1 & 38.6 & 24.1 & 0.2 & 2.565 \\
\hline MP-846 & $\mathrm{Kpp}$ & $37^{\circ} 39^{\prime}$ & $119^{\circ} 22^{\prime}$ & 40.1 & 29.3 & 26.8 & 5.2 & 2.66 \\
\hline
\end{tabular}

ages of the modal constituents are plotted on the simplified geologic maps of figures 4 through 7 . Isopleths have been drawn where the data permit, but no attempt was made to contour all the data systematically. Several of the intrusive sequences, notably those of Washburn Lake and Buena Vista Crest and Tuolumne Intrusive Suite, show systematic variations inward from the margin. The observed trends are an inward decrease in plagioclase and mafic minerals and an inward increase in quartz and potassium feldspar. Similar variations were noted during mapping of the granodiorites of Mount Givens and Jackass Lakes, 


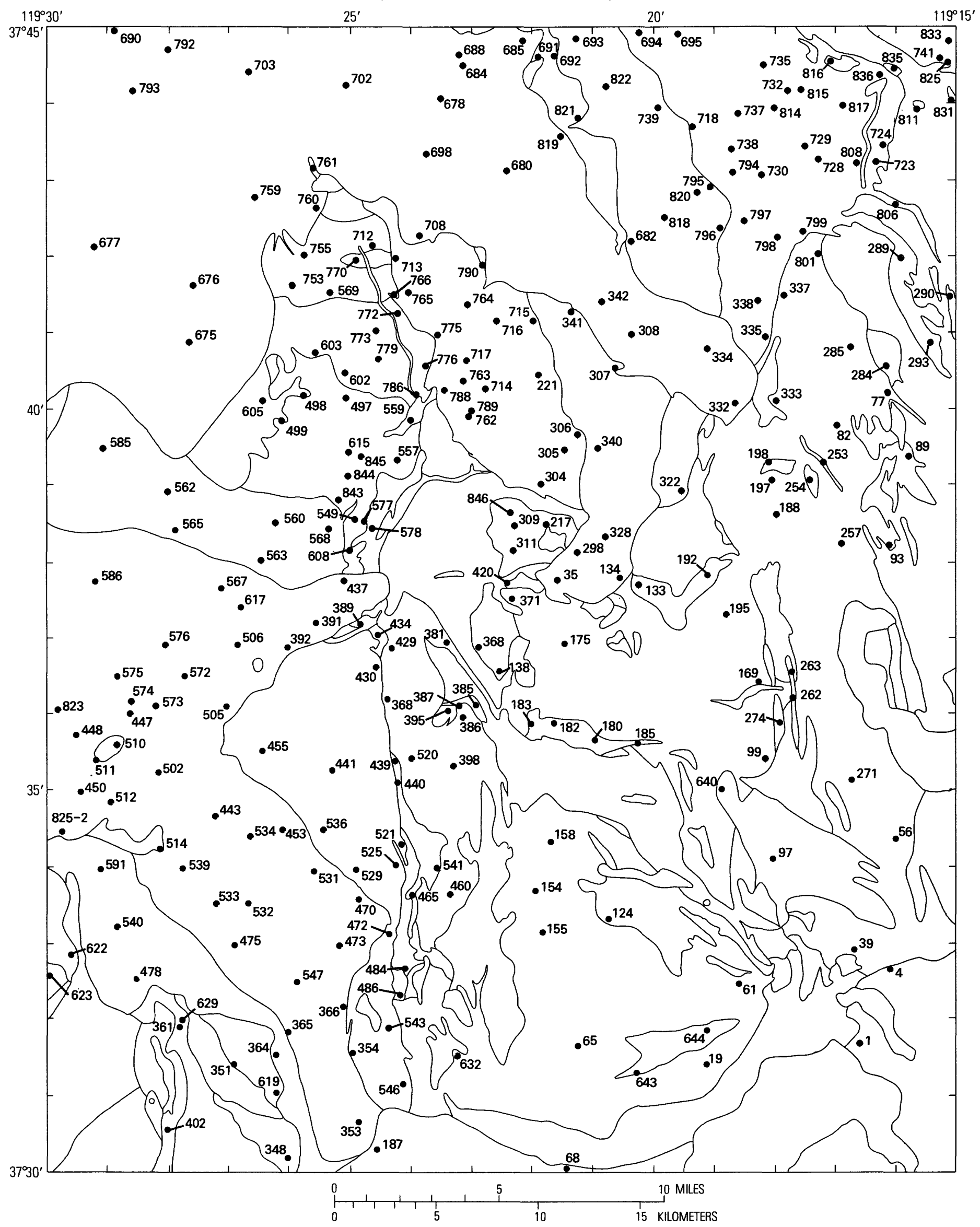

FIGURE 3.-Simplified bedrock geologic map of the Merced Peak quadrangle, showing locations of modally analyzed plutonic rock samples. Explanation in figure 1. 


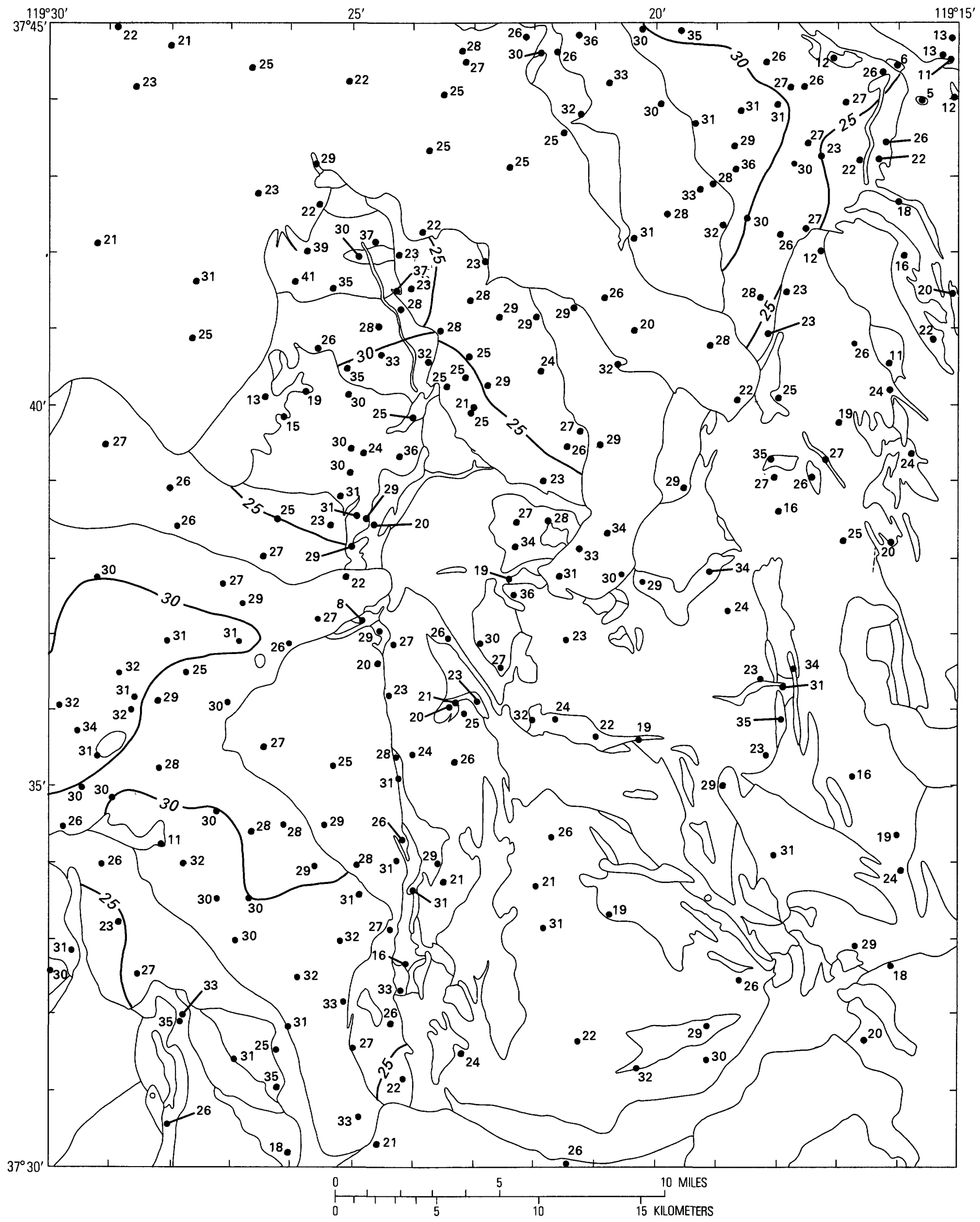

FIGURE 4. - Simplified bedrock geologic map of the Merced Peak quadrangle, showing localities of samples analyzed for quartz in volume percent. Generalized isopleths show equal volume percent. Contour interval 5 percent. Explanation in figure 1. 
MERCED PEAK QUADRANGLE, CENTRAL SIERRA NEVADA, CALIFORNIA-ANALYTIC DATA

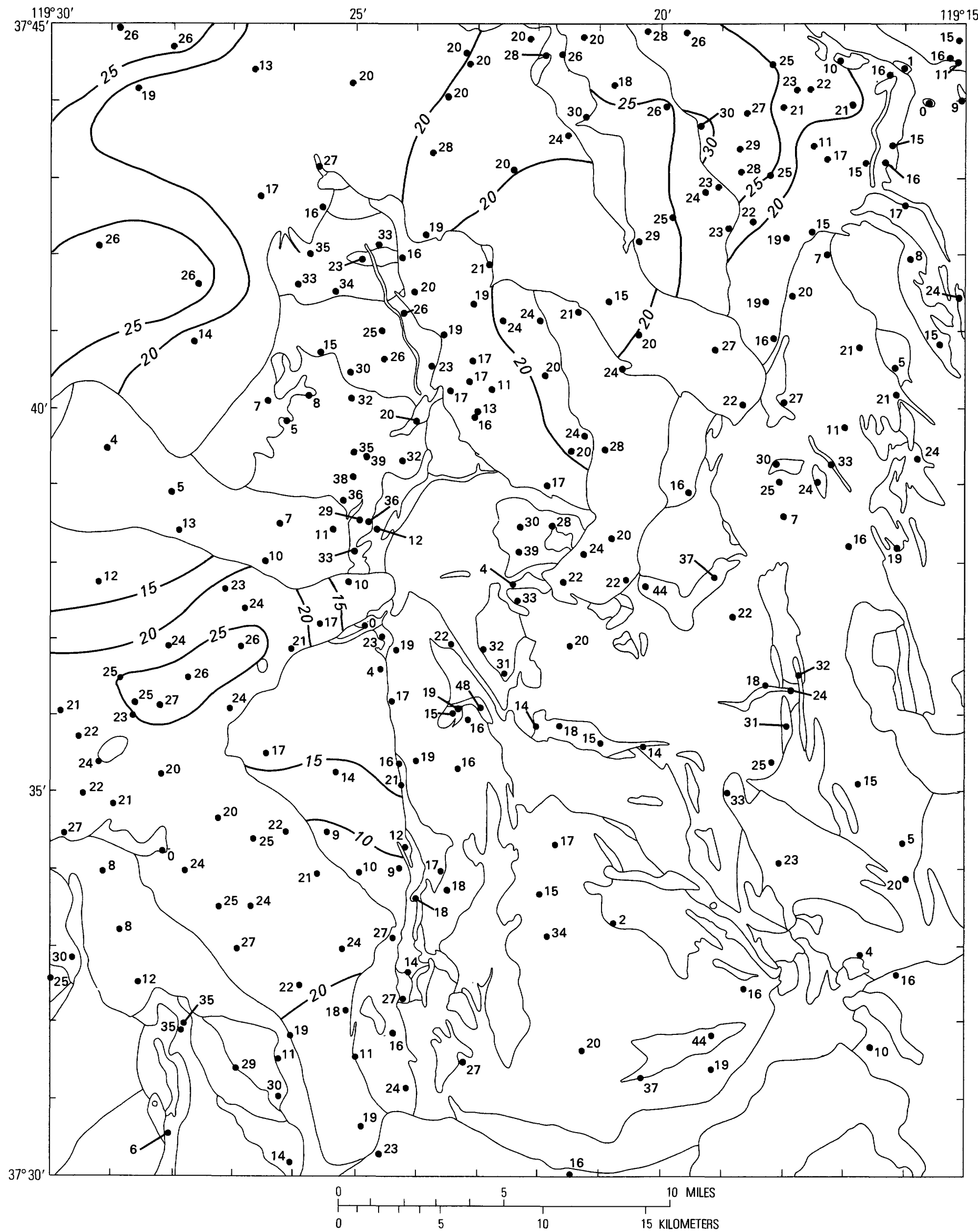

FIGURE 5. - Simplified bedrock geologic map of the Merced Peak quadrangle, showing localities of samples analyzed for potassium feldspar in volume percent. Generalized isopleths show equal volume percent. Contour interval 5 percent. Explanation in figure 1. 


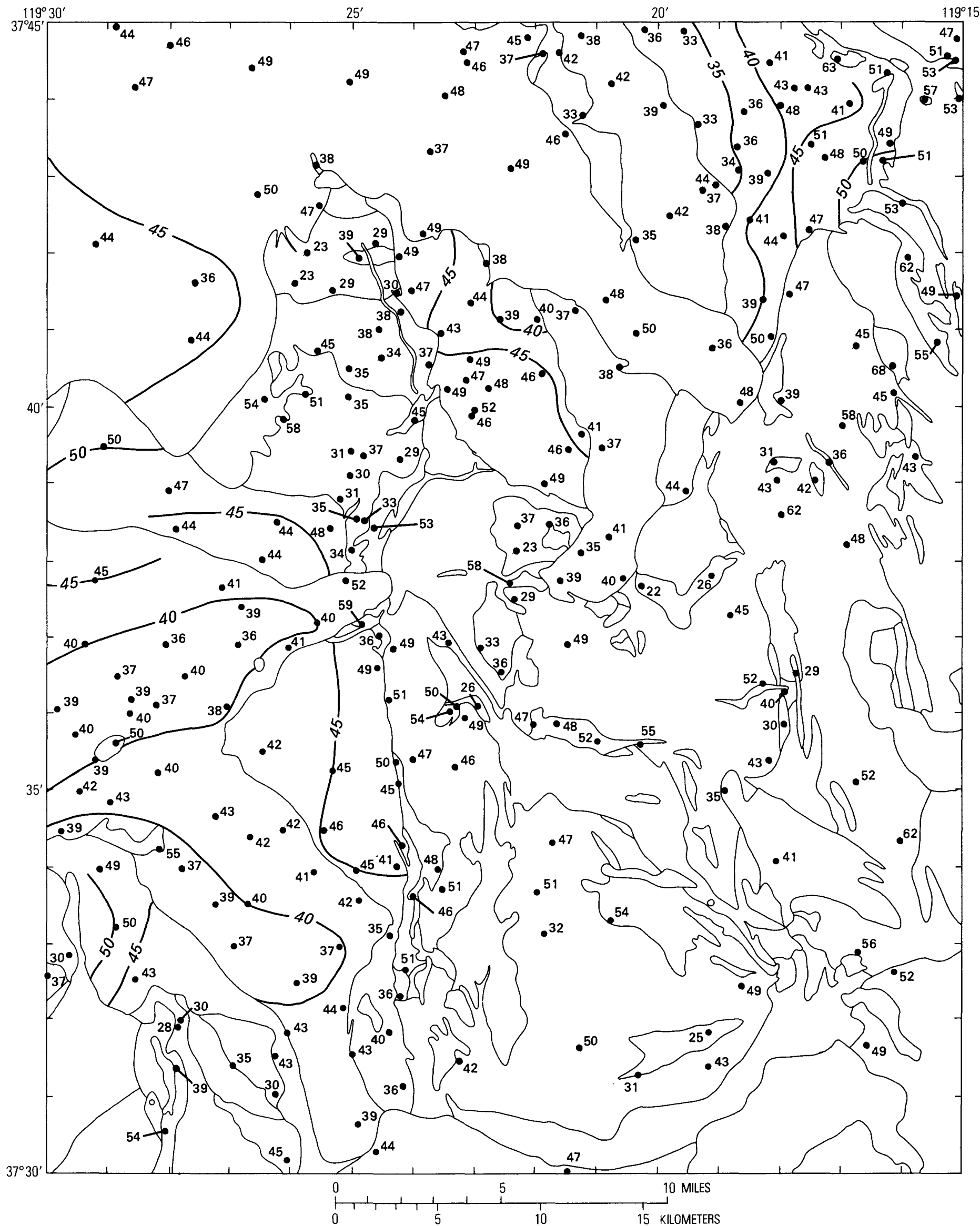

FIGURE 6. - Simplified bedrock geologic map of the Merced Peak quadrangle, showing localities of samples analyzed for plagioclase in volume percent. Generalized isopleths show equal volume percent. Contour interval 5 percent. Explanation in figure 1. 
MERCED PEAK QUADRANGLE, CENTRAL SIERRA NEVADA, CALIFORNIA-ANALYTIC DATA

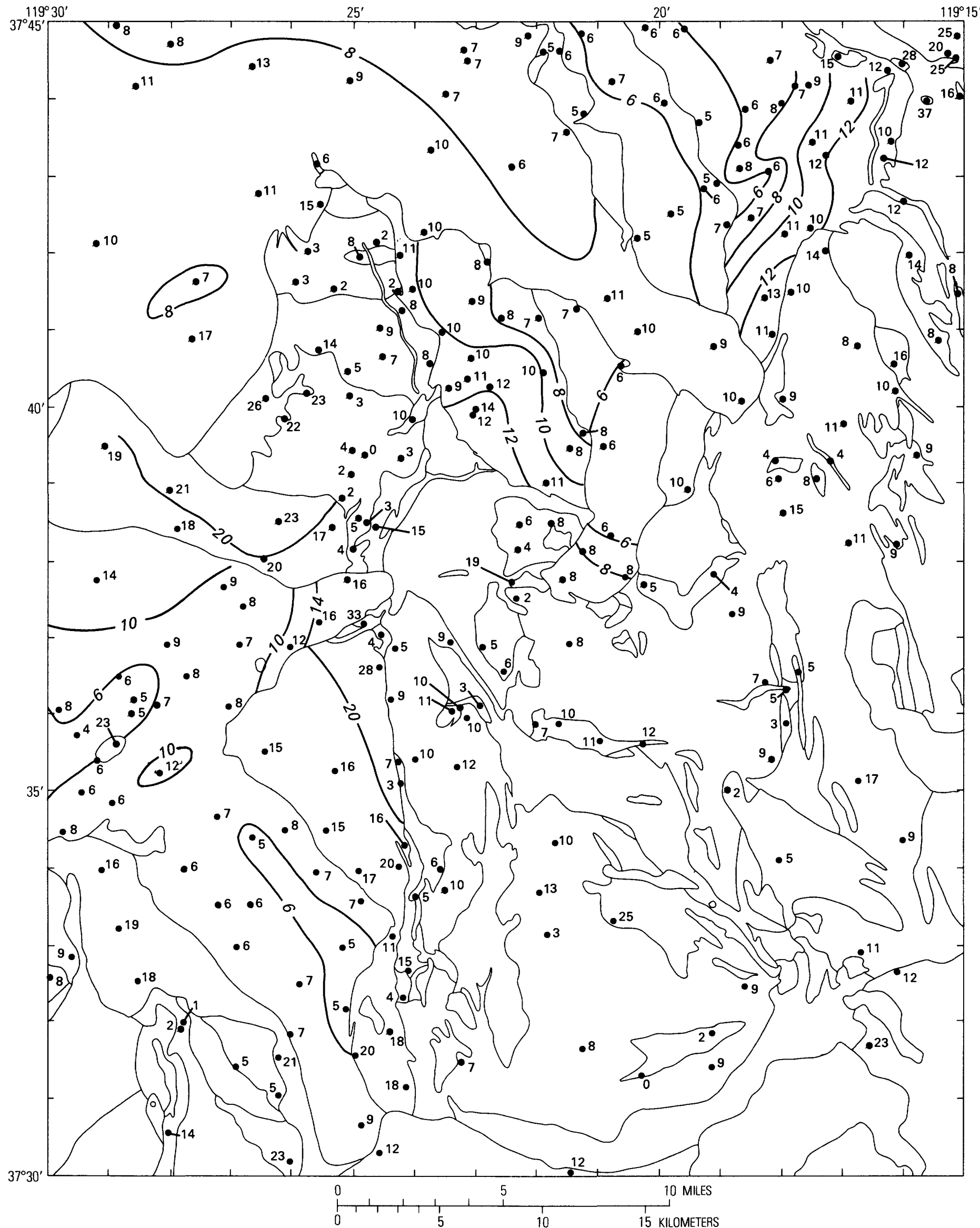

FIGURE 7.-Simplified bedrock geologic map of the Merced Peak quadrangle, showing localities of samples analyzed for mafic minerals in volume percent. Generalized isopleths show equal volume percent. Contour interval 5 percent. Explanation in figure 1. 
but too few samples were collected to document the variations. Several of the plutons, however, do not show systematic modal variations with respect to their margins; this applies particularly to the bodies of quartz diorite and tonalite and to those of leucogranite and leucogranite porphyry.

Biotite, hornblende, and accessory minerals cannot be distinguished consistently on stained slabs, so their separate amounts were not determined with the modes. The relative abundance of biotite and hornblende was determined for many of the chemically analyzed samples by counts of 1,000 to 2,000 points in thin sections, and the results are given in table 4 . In general, biotite and hornblende are in nearly equal abundance in quartz diorite and tonalite, biotite predominates in granodiorite, and biotite occurs to the near exclusion of hornblende in granite.

The composition of plagioclase in most of the chemically analyzed samples was determined by measuring extinction angles of albite twins on the universal stage. The ranges and average compositions so determined are listed in table 4 . In general, the average plagioclase composition of quartz diorite and tonalite ranges from $A n_{35}$ to $A n_{45}$, that of granodiorite from $A n_{25}$ to $A n_{35}$, and that of granite from $A n_{20}$ to $A n_{30}$.

The bulk specific gravities of 412 samples of plutonic and metavolcanic rocks were measured on a directreading balance and are plotted in figure 8 . Those of modally analyzed plutonic rocks are listed in table 5 . Table 4 gives the powder densities of chemically analyzed plutonic and metavolcanic rocks. The intrusive sequences of Washburn Lake and Buena Vista Crest show a systematic decrease in specific gravity inward from the margins, ranging from $2.68 \mathrm{~g} / \mathrm{cc}$ at the margins to $2.64 \mathrm{~g} / \mathrm{cc}$ or less in the cores. In general, quartz diorite and tonalite have a specific gravity of 2.83 to $2.73 \mathrm{~g} / \mathrm{cc}$, granodiorite ranges from 2.73 to $2.66 \mathrm{~g} / \mathrm{cc}$, and granite ranges from 2.66 to $2.63 \mathrm{~g} / \mathrm{cc}$. Plotted specific-gravity values of metavolcanic rocks range from 2.56 to 2.95 $\mathrm{g} / \mathrm{cc}$. The values for metavolcanic rocks along the eastern border of the quadrangle show the largest variation and a high average value of $2.76 \mathrm{~g} / \mathrm{cc}$, reflecting the varied composition of the metavolcanic rocks along the western margin of the Ritter Range pendant and their andesitic average composition. The specific-gravity values of the metavolcanic rocks farther west in the quadrangle (excluding those in the southwest corner) show a more limited range; almost all are between 2.60 and 2.72 and average 2.66 , reflecting the rhyodacitic composition of these metatuffs.

The percentage contents of quartz, potassium feldspar, and plagioclase were recalculated to 100 and plotted on ternary diagrams (figs. $9 B$ through $9 G$.). Ternary compositions mostly lie within the granodiorite and granite fields (fig. $9 \mathrm{~A}$ ) along a band extending from 20 percent quartz, 70 percent plagioclase, and 10 percent orthoclase to 35 percent quartz, 30 percent plagioclase, and 35 percent orthoclase. The intrusive sequence of Buena Vista Crest, however, as well as the granite of Gray Peak, the granodiorite of Grizzly Creek, and El Capitan Granite (as shown by modal analysis of samples from the adjacent Yosemite quadrangle) range along a band of more constant quartz content, from approximately 30 percent quartz, 65 percent plagioclase, and 5 percent orthoclase to 35 percent quartz, 30 percent plagioclase, and 35 percent orthoclase.

The 67 chemical analyses listed in table 4 include 55 prepared by the rapid method of Shapiro and Brannock (1962) and 8 prepared in the U.S. Geological Survey laboratories in Denver using standard methods described by L. C. Peck (1964). The percentage contents of the computer-calculated normative quartz, orthoclase, and plagioclase $(a b+a n)$ of the plutonic and metavolcanic rocks are plotted in figure 10. Faint lines on the triangular plot connect the normative composition of some of the analyzed samples to the modal composition of the same samples in terms of quartz, alkali feldspar, and plagioclase. Modal compositions of most quartz diorite, tonalite, and granodiorite lie closer to the quartz-plagioclase side of the diagram than does the normative plot because the $\mathrm{K}_{2} \mathrm{O}$ content of modal hornblende and biotite is calculated as orthoclase in the norm. Modal compositions of most granites, in contrast, lie farther from the quartz-plagioclase side because albite in the modal perthitic feldspars is counted with potassium feldspar as the alkali feldspar component. Compositions near the granite-granodiorite boundary differ little between normative and modal plots because of the compensating effect of the two factors. 
MERCED PEAK QUADRANGLE, CENTRAL SIERRA NEVADA, CALIFORNIA-ANALYTIC DATA

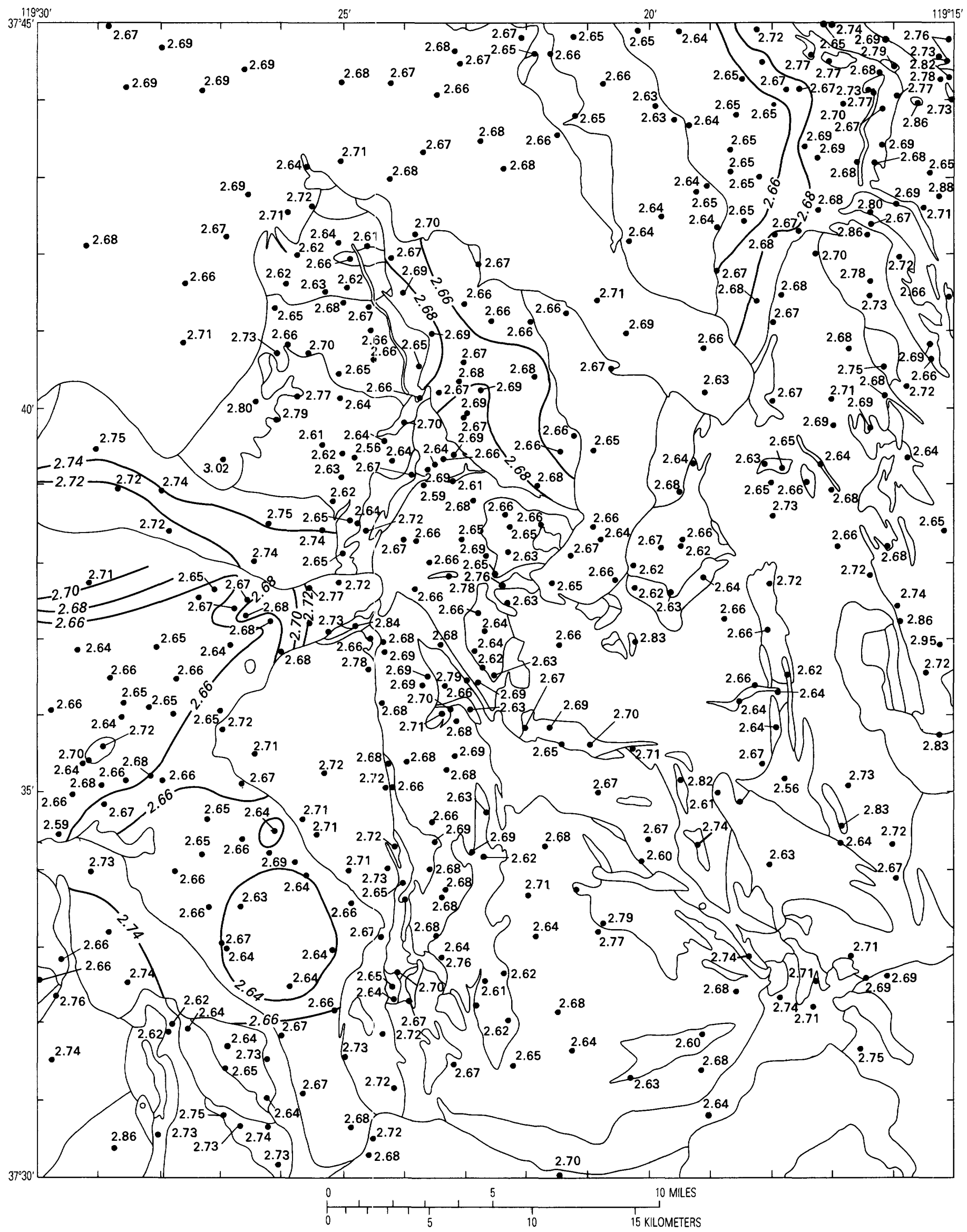

FIGURE 8.-Simplified bedrock geologic map of the Merced Peak quadrangle, showing localities of samples analyzed for specific gravity. Generalized isopleths show equal specific gravity. Contour interval 0.02 percent. Explanation in figure 1. 


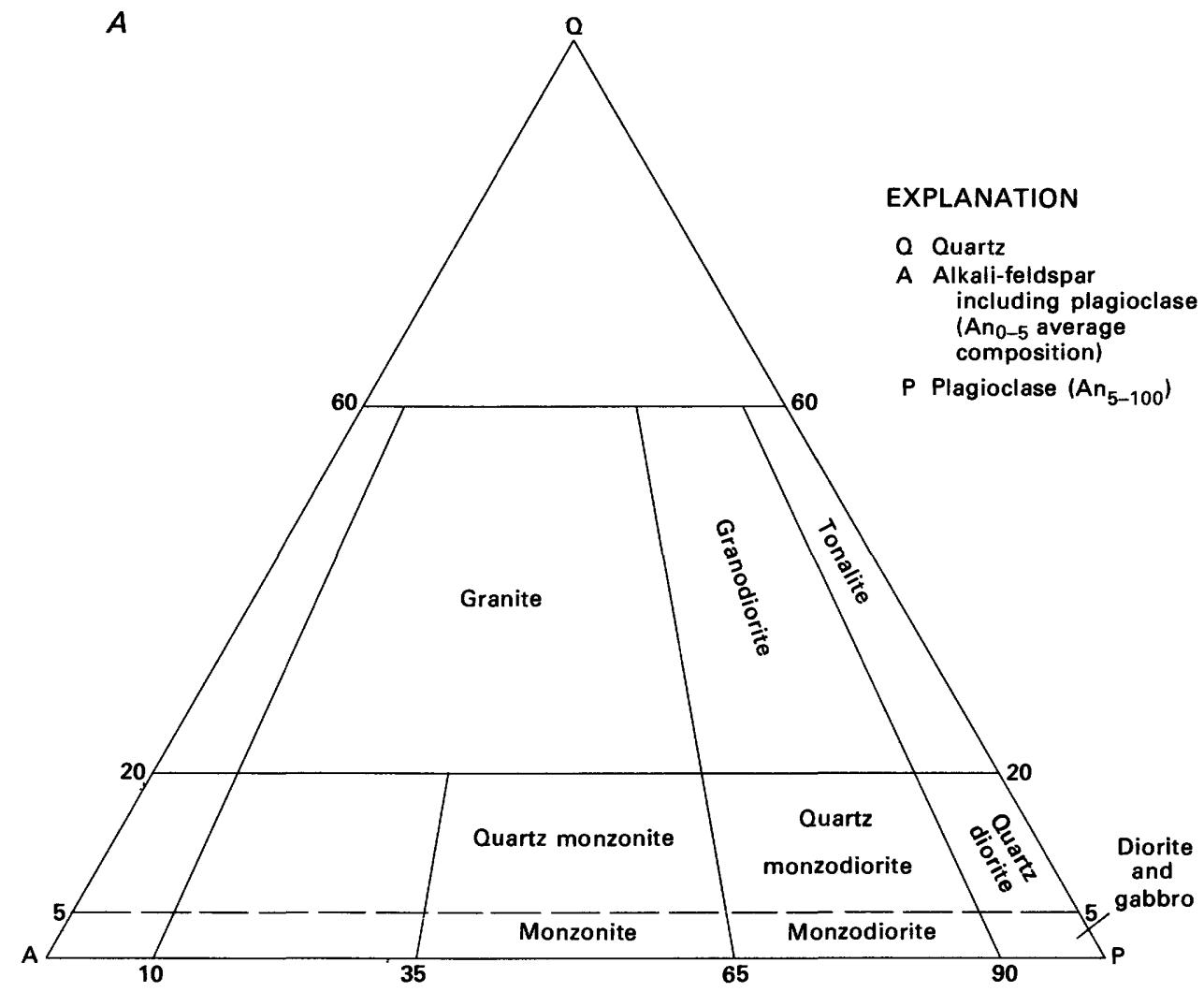

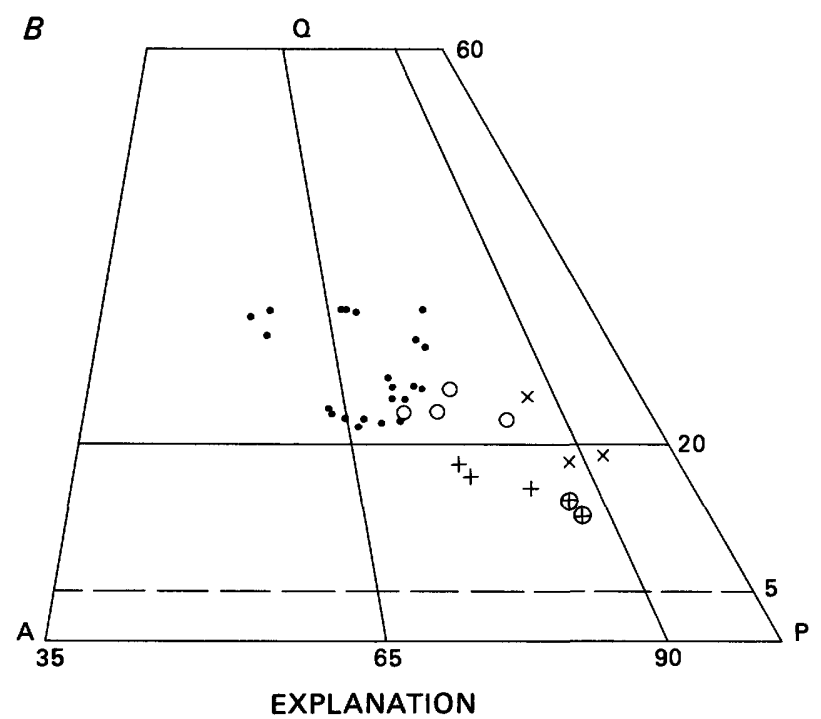

Tuolumne Intrusive Suite

- Half Dome Granodiorite

+ Granodiorite of Kuna Crest

$\times$ Granodiorite of Grayling Lake

$\oplus$ Granodiorite of Kuna Crest

O Mount Givens Granodiorite

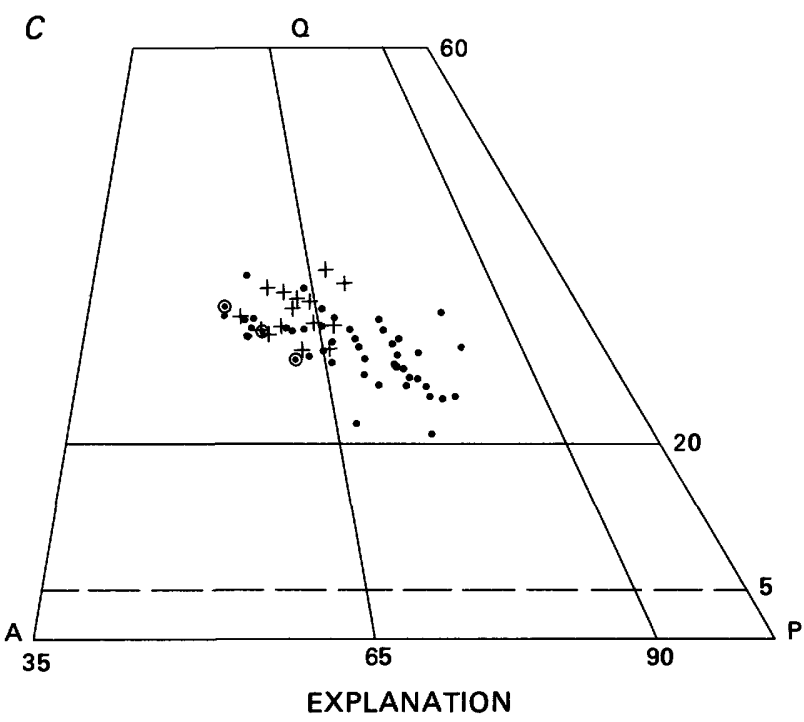

Intrusive sequence of Washburn Lake

- Granite porphyry of Cony Crags

+ Granite of Turner Lake

- Granodiorite of Red Devil Lake 


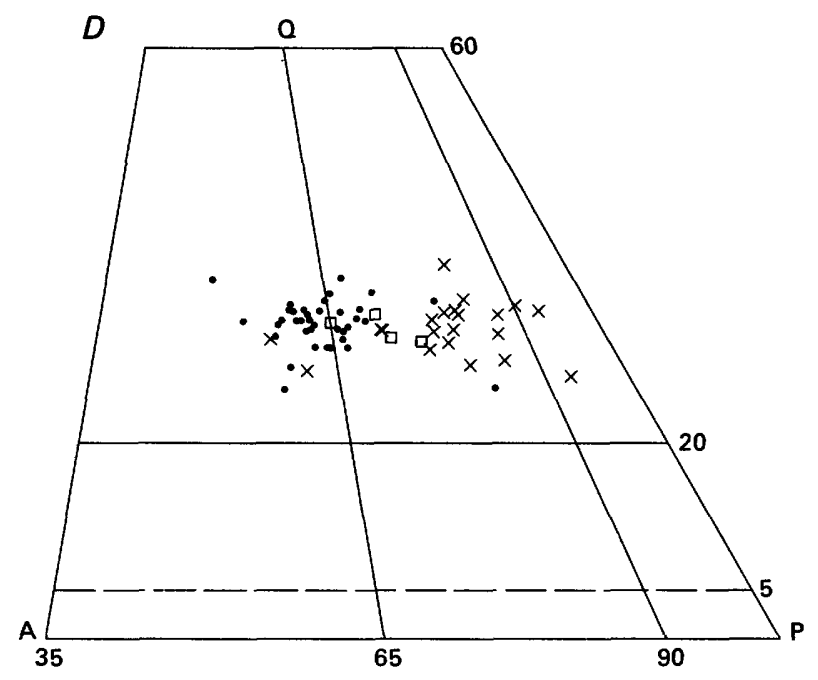

EXPLANATION

Intrusive sequence of Buena Vista Crest

$\square$ Granodiorite of Breeze Lake

- Granodiorite of Ostrander Lake

$x$ Granodiorite of Illilouette Creek

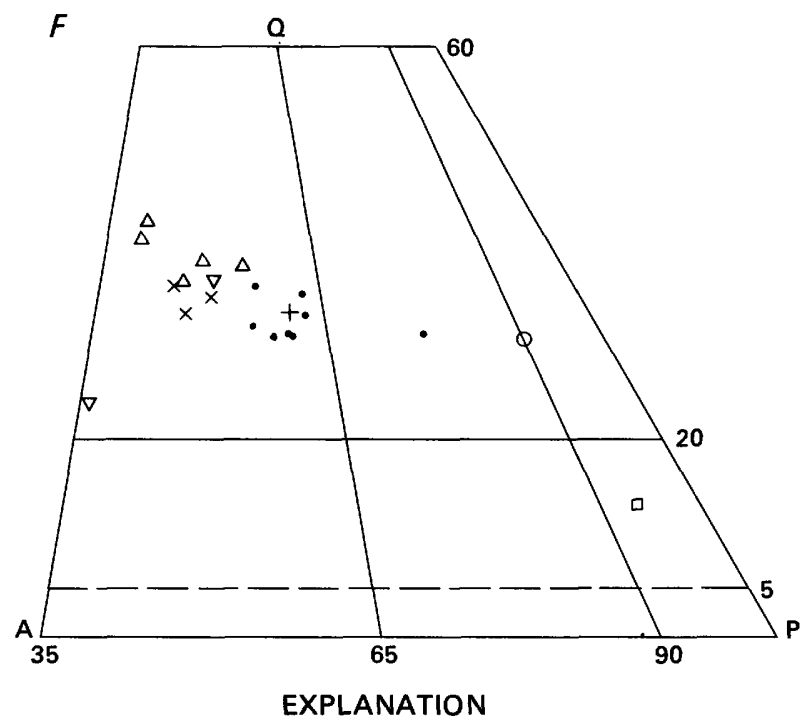

$\nabla$ Leucogranite in small masses

$\square$ Quartz diorite of Long Creek

$\times$ Leucogranite of Shuteye Peak

+ El Capitan Granite

- Granite of Gray Peak

$\Delta$ Leucogranite of Mount Clark

- Granodiorite of Grizzly Creek

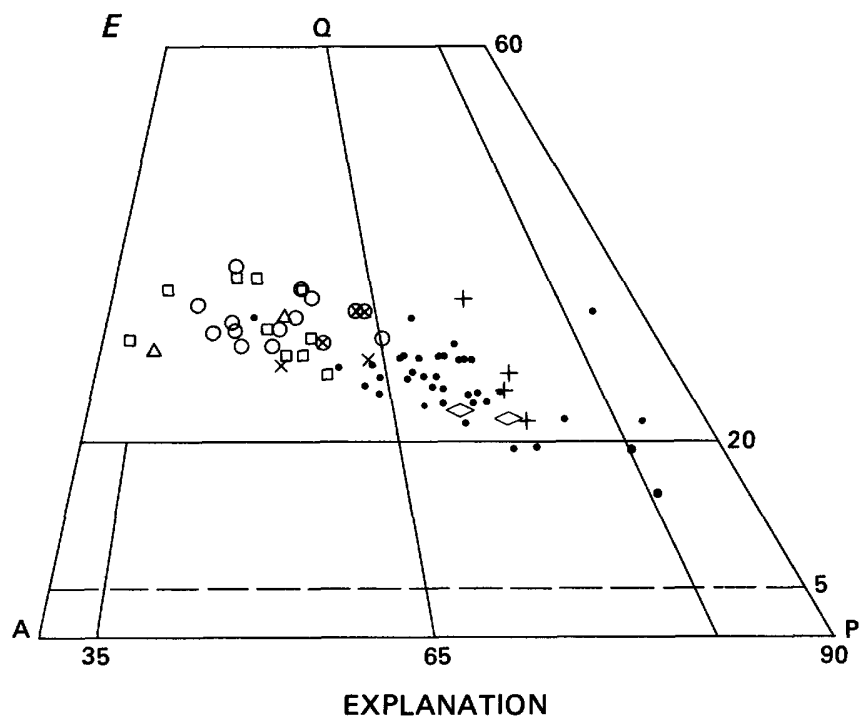

Intrusive sequence of Merced Peak

$\otimes$ Leucogranite of Timber Knob

$\Delta$ Leucogranite of Norris Creek

- Granodiorite of Jackass Lakes

$\times$ Leucogranite phase near

Sadler Peak

+ Granodiorite phase near Ann Lake

- Leucogranite porphyry of Red Peak

$\square$ Leucogranite porphyry of Post Peak

$\diamond$ Granodiorite porphyry of Horsethief Canyon

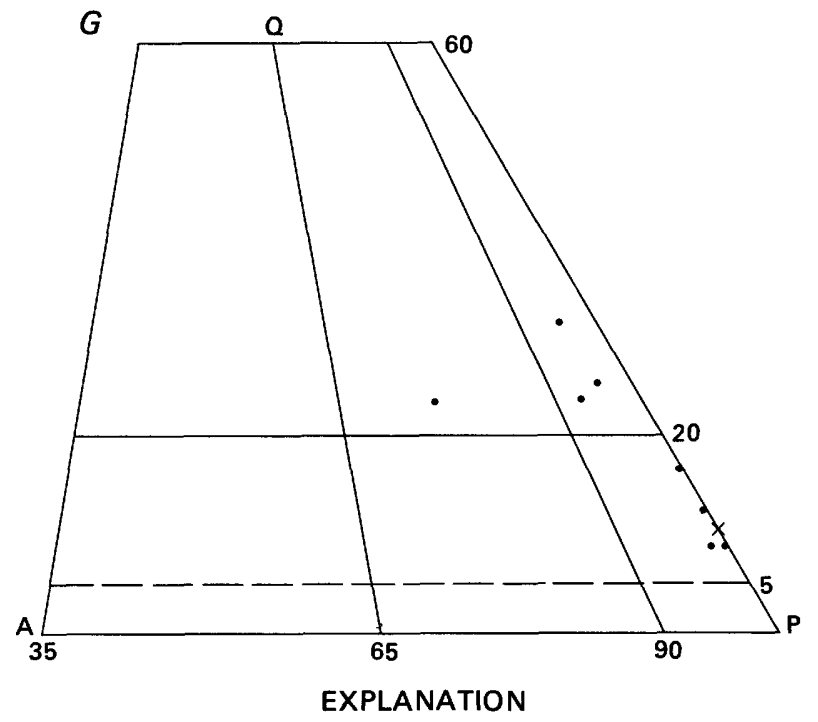

- Quartz diorite and tonalite

$\times$ Microgabbro of the Clark Range

FIGURE 9.-Plots of modes of granitic rocks. Classification plan by Streckeisen and others (1973). $A$, Ternary compositions; $B$, Tuolumne Intrusive Suite; $C$, intrusive sequence of Washburn Lake; $D$, intrusive sequence of Buena Vista Crest; $E$, intrusive sequence of Merced Peak; $F$, other granitic rocks; $G$, quartz diorite, tonalite, and microgabbro. 


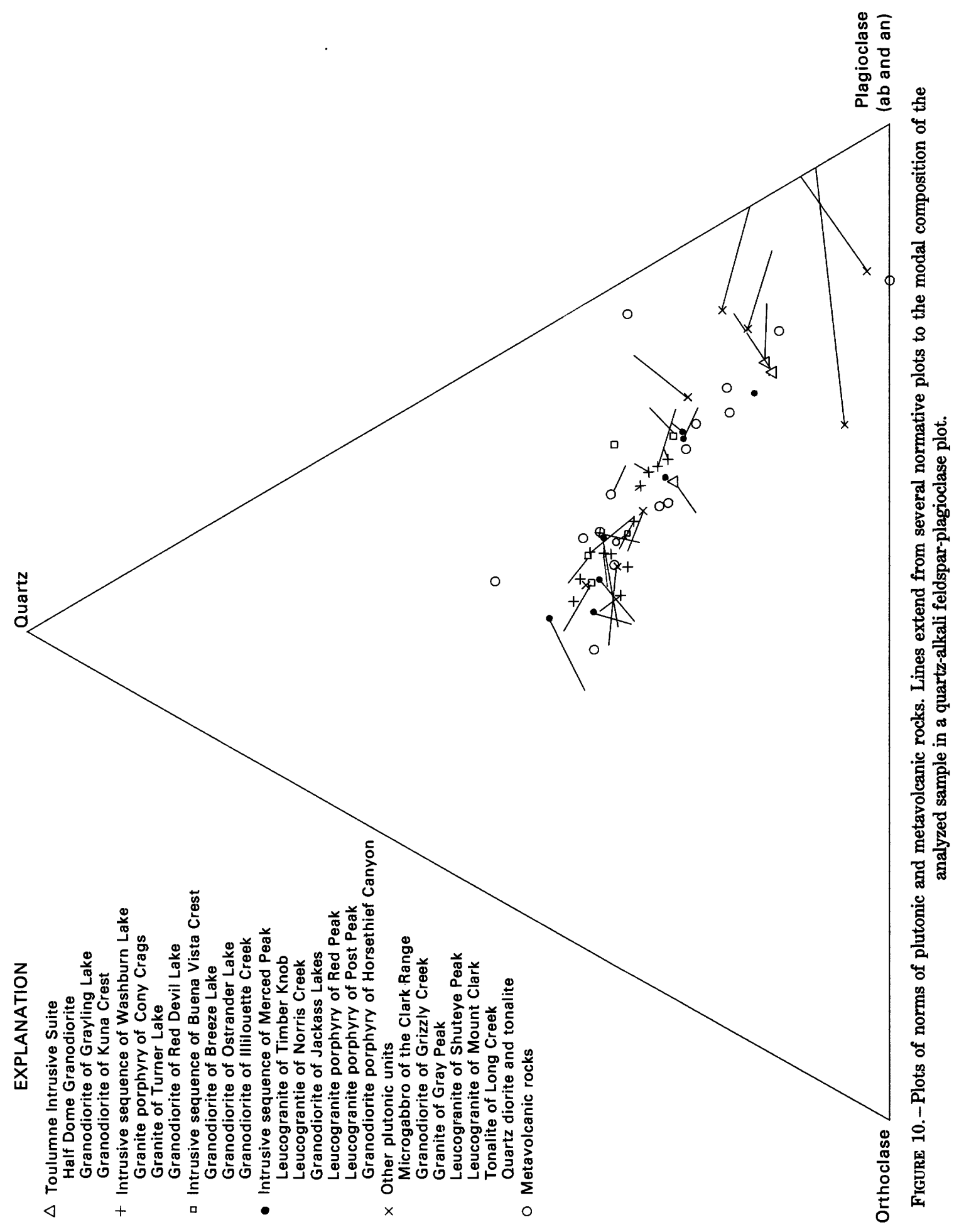




\section{REFERENCES CITED}

Bateman, P. C., Clark, L. D., Huber, N. K., Moore, J. G., and Rinehart, C. D., 1963, The Sierra Nevada batholith-A synthesis of recent work across the central part: U.S. Geological Survey Professional Paper 414-D, $46 \mathrm{p}$.

Bateman, P. C., and Eaton, J. P., 1967, Sierra Nevada batholith: Science, v. 158 , no. 3807 , p. $1407-1417$.

Bateman, P. C., and Lockwood, J. P., 1976, Shaver Lake quadrangle, central Sierra Nevada, California-Analytical data: U.S. Geological Survey Professional Paper 774-D, 20 p.

Bateman, P. C., Lockwood, J. P., and Lydon, P. A., 1971, Geologic map of the Kaiser Peak quadrangle, central Sierra Nevada, California: U.S. Geological Survey Geologic Quadrangle Map GQ-894, scale 1:62,500.

Bateman, P. C., and Wahrhaftig, Clyde, 1966, Geology of the Sierra Nevada, in Bailey, E. H., ed., Geology of northern California: California Division of Mines and Geology Bulletin 190, p. 107-172.

Birman, J. H., 1964, Glacial geology across the crest of the Sierra Nevada, California: Geological Society of America Special Paper $75,80 \mathrm{p}$.

Calkins, F. C., 1930, The granitic rocks of the Yosemite region, in Matthes, F. E., ed., Geologic history of the Yosemite Valley: U.S. Geological Survey Professional Paper 160, p. 120-129.

Carmichael, I. S. E., 1967, The mineralogy and petrology of the volcanic rocks from the Leucite Hills, Wyoming: Contributions to Mineralogy and Petrology, v. 15, no. 1, p. 24-66.

Dalrymple, G. B., 1963, Potassium-argon dates of some Cenozoic volcanic rocks of the Sierra Nevada, California: Geological Society of America Bulletin, v. 74, no. 4, p. 379-390.

1964, Cenozoic chronology of the Sierra Nevada, California: California University Publications in Geological Sciences, v. 47, $41 \mathrm{p}$.

Hamilton, W. B., and Neuerburg, G. J., 1956, Olivine-sanidine trachybasalt from the Sierra Nevada, California: American Mineralogist, v. 41 , nos. $11-12$, p. 851-873.

Huber, N. K., 1968, Geologic map of the Shuteye Peak quadrangle, Sierra Nevada, California: U.S. Geological Survey Geologic Quadrangle Map GQ-728, scale 1:62,500.

Huber, N. K., and Rinehart, C. D., 1965, Geologic map of the Devils Postpile quadrangle, Sierra Nevada, California: U.S. Geological Survey Geologic Quadrangle Map GQ-437, scale 1:62,500.

1967, Cenozoic volcanic rocks of the Devils Postpile quadrangle, eastern Sierra Nevada, California: U.S. Geological Survey Professional Paper 554-D, 21 p.

Jones, D. L., and Moore, J. G., 1973, Lower Jurassic ammonite from the south-central Sierra Nevada, California: U.S. Geological Survey Journal of Research, v. 1, no. 4, p. 453-458.

Kistler, R. W., 1966a, Geologic map of the Mono Craters quadrangle, Mono and Tuolumne counties, California: U.S. Geological Survey Geologic Quadrangle Map GQ-462.

1966b, Structure and metamorphism in the Mono Craters quad- rangle, Sierra Nevada, California: U.S. Geological Survey Bulletin 1221-E, $53 \mathrm{p}$.

1973, Geologic map of the Hetch Hetchy Reservoir quadrangle,

Yosemite National Park, California: U.S. Geological Survey Geologic Quadrangle Map GQ-1112, scale 1:62,500.

1974, Hetch Hetchy Reservoir quadrangle, Yosemite National Park, California-Analytic data: U.S. Geological Survey Professional paper 774-B, $15 \mathrm{p}$.

Matthes, F. E., 1930, Geologic history of the Yosemite Valley: U.S. Geological Survey Professional Paper 160, 137 p.

1960, Reconnaissance of the geomorphology and glacial geology of the San Joaquin basin, Sierra Nevada, California: U.S. Geological Survey Professional Paper 329, $62 \mathrm{p}$.

Nokleberg, W. J., 1970, Geology of the Strawberry Mine roof pendant, central Sierra Nevada, California: Santa Barbara, University of California at Santa Barbara, Ph.D. dissertation, 157 p.

1981, Stratigraphy and structure of the Strawberry Mine roof pendant, central Sierra Nevada, California: U.S. Geological Survey Professional Paper 1154, 18 p.

Norman, M. B., 1974, Improved techniques for selective staining of feldspar and other minerals using amaranth: U.S. Geological Survey Journal of Research, v. 2, no. 1 p. 73-79.

Peck, D. L., 1964, Preliminary geologic map of the Merced Peak quadrangle, California: U.S. Geological Survey Mineral Investigations Field Studies Map MF-281, scale 1:48,000.

1980, Geologic map of the Merced Peak quadrangle, central Sierra Nevada, California: U.S. Geological Survey Geologic Quadrangle Map GQ-1531, scale 1:62,500.

Peck, L. C., 1964, Systematic analysis of silicates: U.S. Geological Survey Bulletin $1170,89 \mathrm{p}$.

Shapiro, L., 1975, Rapid analysis of silicate, carbonate, and phosphate rocks-Revised edition: U.S. Geological Survey Bulletin 1401, $76 \mathrm{p}$.

Shapiro, Leonard, and Brannock, W. W., 1962, Rapid analyses of silicate, carbonate, and phosphate rocks: U.S. Geological Survey Bulletin 1144-A, 56 p.

Streckeisen, A. L., and others, 1973, Plutonic rocks-classification and nomenclature recommended by the IUGS Subcommission on the Systematics of Igneous Rocks: Geotimes, v. 18, no. 10, p. 26-30.

Stern, T. W., Bateman, P. C., Morgan, B. A., Newell, M. F., and Peck, D. L., 1981, Isotopic U-Pb ages of zircon from the granitoids of the central Sierra Nevada, California: U.S. Geological Survey Professional Paper 1185, $17 \mathrm{p}$.

Van Kooten, G. K., 1980, Mineralogy, petrology, and geochemistry of an ultrapotassic basaltic suite, central Sierra Nevada, California, U.S.A.: Journal of Petrology, v. 21, no. 4, p. 651-684.

Williams, Howel, 1936, Pliocene volcanoes of the Navajo-Hopi County [Arizona]: Geological Society of America Bulletin, v. 47, no. 1, p. 111-172.

York, Derek, 1966, Least-squares fitting of a straight line: Canadian Journal of Physics, v. 44, p. 1079-1086. 